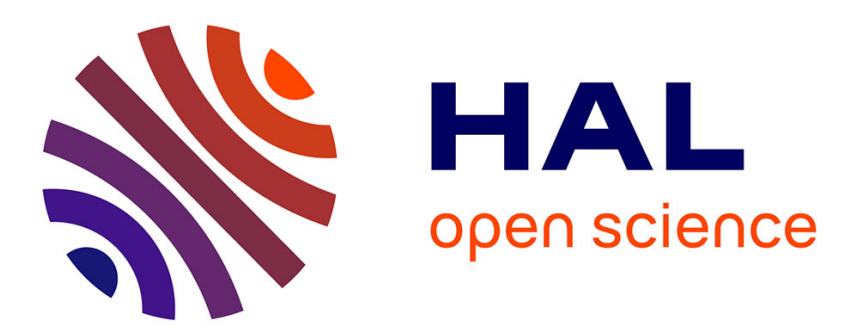

\title{
Leveraging Live Machine Learning and Deep Sleep to Support a Self-Adaptive Efficient Configuration of Battery Powered Sensors
}

Cyril Cecchinel, François Fouquet, Sébastien Mosser, Philippe Collet

\section{- To cite this version:}

Cyril Cecchinel, François Fouquet, Sébastien Mosser, Philippe Collet. Leveraging Live Machine Learning and Deep Sleep to Support a Self-Adaptive Efficient Configuration of Battery Powered Sensors. Future Generation Computer Systems, In press. hal-01880430

\section{HAL Id: hal-01880430 https://hal.science/hal-01880430}

Submitted on 24 Sep 2018

HAL is a multi-disciplinary open access archive for the deposit and dissemination of scientific research documents, whether they are published or not. The documents may come from teaching and research institutions in France or abroad, or from public or private research centers.
L'archive ouverte pluridisciplinaire HAL, est destinée au dépôt et à la diffusion de documents scientifiques de niveau recherche, publiés ou non, émanant des établissements d'enseignement et de recherche français ou étrangers, des laboratoires publics ou privés. 


\title{
Leveraging Live Machine Learning and Deep Sleep to Support a Self-Adaptive Efficient Configuration of Battery Powered Sensors
}

\author{
Cyril Cecchinel, \\ François Fouquet ${ }^{1}$ \\ DataThings, Luxembourg \\ Sébastien Mosser, \\ Philippe Collet ${ }^{2}$ \\ Université Côte d'Azur, CNRS, I3S, France
}

\begin{abstract}
Sensor networks empower Internet of Things (IoT) applications by connecting them to physical world measurements. However, the necessary use of limited bandwidth networks and battery-powered devices makes their optimal configuration challenging. An over-usage of periodic sensors (i.e. too frequent measurements) may easily lead to network congestion or battery drain effects, and conversely, a lower usage is likely to cause poor measurement quality. In this paper we propose a middleware that continuously generates and exposes to the sensor network an energy-efficient sensors configuration based on data live observations. Using a live learning process, our contributions dynamically act on two configuration points: (i) sensors sampling frequency, which is optimized based on machine-learning predictability from previous measurements, (ii) network usage optimization according to the frequency of requests from deployed software applications. As a major outcome, we obtain a self-adaptive platform with an extended sensors battery life while ensuring a proper level of data quality and freshness. Through theoretical and experimental assessments, we demonstrate the capacity of our approach to constantly find a near-optimal tradeoff between sensors and network usage, and measurement quality. In our experimental validation, we have successfully scaled up the battery lifetime of a temperature sensor from a monthly to a yearly basis.
\end{abstract}

\footnotetext{
1 \{cyril.cecchinel,francois.fouquet\}@datathings.com

$2\{$ mosser,collet $\} @ i 3 s . u n i c e . f r$
} 


\section{Introduction}

The most recent Gartner group's forecasts predict up to 26 billions of things that could be connected to the Internet by 2020 forming the Internet of Things (IoT). Once measured, data gathered from IoT sensors are key enablers for next generation infrastructures such as Smart Cities or Smart Grid.

In sensing infrastructures [1], sensor networks are responsible for measuring physical phenomena and sending values to a remote IoT middleware where the collected data can be retrieved by third-party applications. In addition, such middleware is also responsible for managing the devices deployed in the sensor networks [2]. In particular, the middleware defines how the data is retrieved from the sensor network either using pulling or pushing mechanisms.

Using a pulling approach implies sensors to be always reachable but sensor networks are mainly relying on battery-powered devices and limited bandwidth networks. Due to these constraints, IoT devices are most of the time not directly reachable and need to adopt a push mechanism to share the measured data with a remote middleware. Regardless of the network technology used, the configuration of this push mechanism directly impacts resources usage, as well as the chosen measurement frequency. In a nutshell, a sensor loops over a very simple and generic process that consists in measuring, buffering, pushing data, sleeping and looping again. During measuring, pushing and sleeping phases, sensors have drastically different battery usages, from milliamp to microamp per hour, as shown by theoretical simulations [3] and empirical validations [4]. In addition, last generation microcontrollers offer much more powerful and easy access deepsleep modes thanks to the embedded network layers within the chip [5], which can be activated by disconnecting every unnecessary elements such as network chips between two measurements. Usage of deep sleep can shift the battery life of a sensor [6], from days to years! This makes deep sleep the major tool to design energy-efficient protocols [7], relying for instance on sensor network topology [8]. However, under deep sleep, sensors are unreachable and potentially can miss important measurements. Therefore, using deep sleep should be subject to a tradeoff between data quality and the battery life as a short deepsleep allows the sensors to send data more frequently but drains the battery whereas a long deep-sleep saves the battery but prevents the measurement of values.

Moreover, using fixed period data collection is not efficient as, due to lack of foreseen precise data usage, many IoT infrastructures oversample their sensors. This produces sub-efficient datasets and drain devices' battery. In addition, measured data can be very variable so that a static collection policy will be always sub-efficient. For instance, in a steady state, temperature of a room will be stable and does not require high frequency measurements. Conversely, in heating mode, temperature is less predictable and should be sampled with

higher frequency. There are already many machine learning algorithms allowing the construction of predictive models. As an example, considering a public open 
dataset of temperature sensors ${ }^{3}$, more than $90 \%$ of data are redundant as they could have been extrapolated from a prediction model. This advocates for an adaptive strategy $[7,9]$ that, like cloud computing, adapts resource usage based on observed need [10]. Assuming these data were produced from a batterypowered sensor, it would have over-consumed energy.

We claim that an adaptive strategy could reduce this energy loss, significantly extending the lifetime of the most frequently used batteries in sensor networks. Examples to support this assumption are given in SEC. 3. Implementing this vision, we present here a self-adaptive and energy efficient sensor network middleware driven by machine learning. This contribution aims at reducing the amount of measured data by sensors in order to reduce the battery consumption of them. To do so, we rely on the predictability property of each sensor signal, calculated by a machine learning algorithm. Overall, our middleware relies on learning techniques to compute at any moment the appropriate sampling and pushing period for each connected sensor. These data collection periods are sent to sensors, allowing them to dynamically reconfigure their deep sleep and push periods. To mitigate the risk of missing important measures, deep sleep periods are bounded by a maximum time deviation limit defined by domain experts.

Our contribution also aims at measuring the gain of battery lifetime using such adaptive periods. These experiments were conducted on a prototyping platform running on an ESP8266 architecture powered by a lithium-ion battery. First, we have determined extreme boundaries of microcontroller working conditions by identifying the cutoff voltage (i.e., the minimum voltage at which the platform is still working). Secondly, we have evaluated with fixed sampling periods and adaptive periods the time to reach this cutoff voltage. Then, we have have applied the proposed approach and compare the gain in battery lifetime. Experiments were conducted over weeks while accelerating their usage by artificially reducing the sampling period. Nonetheless, extrapolated results show that our adaptive usage of deep-sleep is solely able to extend the battery lifetime from a weekly basis to a one of many months.

In the following, SEC. 2 presents how one can apply live machine learning techniques upon battery-powered sensor to predict futures values. SEC. 3 supports our assumptions by illustrating the need of adaptive periods based on realistic observations. Then, SEC. 4 focuses on the computation of the appropriate periods while SEC. 5 describes the assessment of the proposed approach on a real platform by observing the battery life-time. Finally, SEC. 7 discusses future work and concludes this article.

\footnotetext{
${ }^{3}$ https://github.com/ulrich06/greycat_senso/blob/master/assets/TEMP_CAMPUS_15d. json
} 


\section{Live machine learning and predictability applied to battery-powered sensors}

In this paper we focus on battery-powered sensors for which energy consumption is a key criterion to ensure their viability in the long run. To reach their energy efficient goal, such devices are usually designed around a microcontroller architecture such as the widely used ESP8266 [11] manufactured by Espressif Systems. The data-sheet of such hardware highlights the tremendous difference of energy consumption according to the use or the absence of use of elements such as network or measurement devices (e.g., thermo-resistance or wifi chip). In others words, the energy consumption is directly related to the sampling and the sending of measurements. As mentioned in the introduction, a mode called deep-sleep can be activated to drastically reduce energy consumption by stopping most of sensor services. As a result the frequency of measurement, network push and deep-sleep activation will have a direct impact on sensor lifespan.

Most of the physical phenomena under measure are inescapably variable. As an example, if a measured temperature is stable, high frequency measurement will waste energy while not participating more to the knowledge acquired about temperature. Based on the variability of physical phenomena and the impact of sampling and sending over the sensor's battery, this work relies on the following general hypothesis $\mathcal{H}$ : "a fixed sampling and sending of measurements generates redundant data and therefore a waste of energy".

Live machine learning techniques help to predict data by discovering a model against a dataset and to reduce the energy consumption by reasoning on this model instead of querying new values from the sensors [12]. In the field of statistics, the regression methods, e.g., the linear regression method, allow one to predict one variable from other variables. This type of regression is built from a fixed set of data. If all the data are not known in advance, the regression methods must be updated with the new data. Such updatable methods are known as autoregressive methods. They allow a compact representation of temporal series datasets [13] as they rely on data predictability to recursively try to replace a range of data by a compact model. Usually, the expected outcome is a compression or simplification of data to enhance later processing. For instance, a polynomial function can replace a range of data by its ability to produce within a tolerated error the initial data points. An autoregressive method can then consist at fitting a polynomial function to this range of data.

Our contribution heavily relies on such techniques and data predictability principle, but for another purpose: we use the predictability principle not for a posteriori compression, but as an input to optimize physical sensor sampling rate. In other words, we want to use an a priori compression to reduce sensor usage.

In addition, autoregressive methods can be used online, while measurements are streamed from sensors [14]. The benefit is an immediate feedback of the learning method, able to be used immediately. However, this live usage implies a computation cost for every input datum, making the ratio between its efficiency and effectiveness critical. 


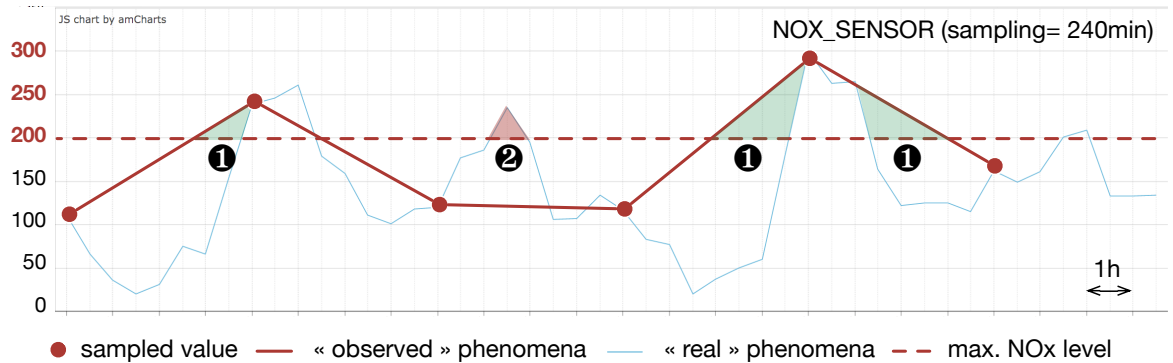

Figure 1: Sampling using a fixed period

To illustrate this tradeoff, we compare a regression method using a lot of buffered data to detect a trend and offer better compression, with an approach without memory which tries to segment data in motion. Due to our live usage with sensors, the performance is key for our approach. For this reason, we rely on polynomial regression model without memory which gives the fastest results according to the tested datasets.

We also note that there are network access protocols designed for batterypowered platforms, but these optimisations are done for mesh networks and are not efficient for client/server architectures promoted by the IoT and IP network everwhere paradigms. We discuss them in section SEC. 6.

\section{On the positive influence of automated sampling on power con- sumption}

In this section, we discuss how live machine learning techniques can help to predict data and we illustrate the gains of using dynamic sampling and sending periods based on our hypothesis $\mathcal{H}$.

\subsection{Impact of automatic sampling period configuration based on sensor pre- dictability}

Data obtained from the sensor network are collected according to a collection period. The definition of a fixed collection period leads to important values being missed between two data sampling. For example, FIG. 1 depicts the values produced by a $N O_{x}$ sensor deployed in an Italian city [15]. The European Union ${ }^{4}$ expects $N O_{x}$ hourly average concentrations to be lower than $200 \mu \mathrm{g} / \mathrm{m}^{3}$ or yearly average concentrations to be lower than $40 \mu \mathrm{g} / \mathrm{m}^{3}$. An alert must be triggered for the inhabitants if these values are exceeded. The sampled values (big dots) obtained from this sensor mislead domain experts. Indeed, with its static collection period, a pollution event is triggered when the pollution is still

\footnotetext{
${ }^{4}$ http://ec.europa.eu/environment/air/quality/standards.htm
} 


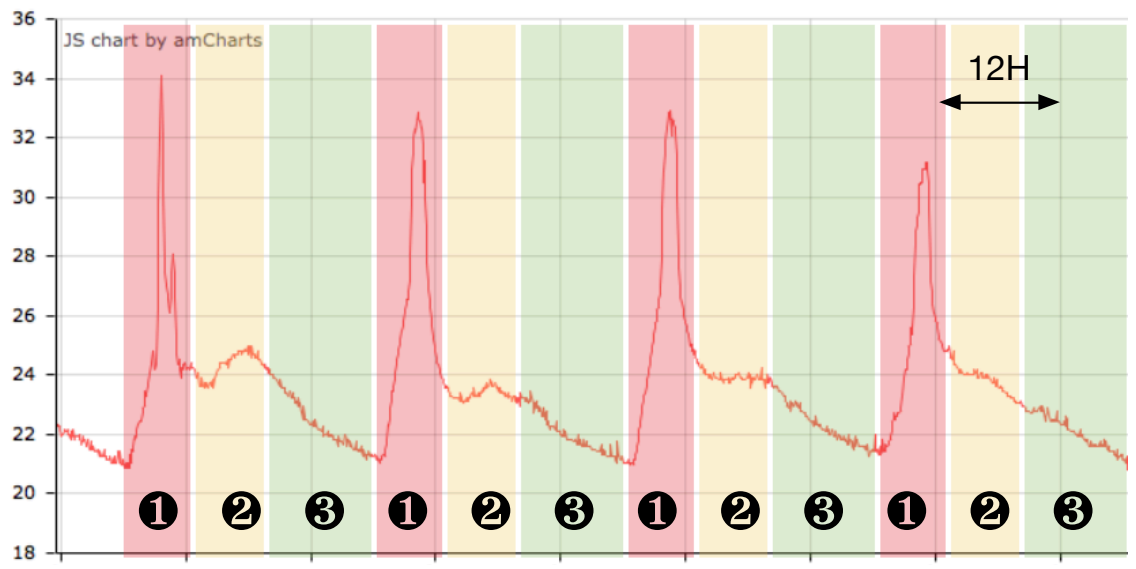

Figure 2: Periodic patterns for a temperature sensor

lower than the threshold value or stays active when the pollution episode is over (areas marked with on FIG. 1), resulting in false alerts. Moreover, some episodes of pollution can be unnoticed (2 areas on FIG. 1), which clearly breaks the law.

In addition, a fixed collection period is irrelevant when observed phenomena follow periodic patterns. Fig. 2 presents temperature data collected from a sensor located in an office exposed to direct morning sunlight. The analysis of data using a one-day window highlights a recurrent three-stages pattern: (i) the temperature first rises quickly (sun hitting the windows) before dropping sharply due to the activation of the air conditioning in the morning (1 on FIg. 2), (ii) then the temperature fluctuates slowly during the day after occupants arrival, as they prefer to switch off the air conditioning and open the window in the afternoon (2) on Fig. 2) and (iii) finally, it decreases smoothly over the night (3) on Fig. 2). As temperature data is varying tremendously during the first stage, a short period of collection is required to reduce the loss. Regarding the second stage, a longer collection period is preferable as values fluctuate slowly. Finally, during the third stage, the collection period can be significantly reduced as values decrease in a linear manner.

In such context, the predictability of physical phenomena can be exploited to increase the data collection period, and thus to lower the energy consumption by reducing the duty cycle and (energy intensive) accesses to the network.

Table ТАВ. 1 presents several public datasets obtained from real measurements. Temperature, light and power consumption values are obtained from sensors deployed on a campus located in southern France ${ }^{5}[16]$. The pollution

\footnotetext{
${ }^{5}$ Temperature and light datasets can be downloaded at https://goo.gl/FWhusm
} 
Table 1: Datasets from real world experiments (campus [16], pollution [15])

\begin{tabular}{lccc}
\hline \multicolumn{1}{c}{ Sensor } & Phenomena & Size (\# measures) & Size (\# days) \\
\hline CAMPUS_TEMP & Temperature & 3823 & 15 \\
OFFICE_LIGHT & Light & 3187 & 15 \\
OFFICE_TEMP & Temperature & 3186 & 15 \\
CO_SENSOR & CO pollution & 9356 & 365 \\
NOX__SENSOR & NOx pollution & 9356 & 365 \\
O3_SENSOR & O3 pollution & 9356 & 365 \\
NO2_SENSOR & NO2 pollution & 9356 & 365 \\
\hline
\end{tabular}

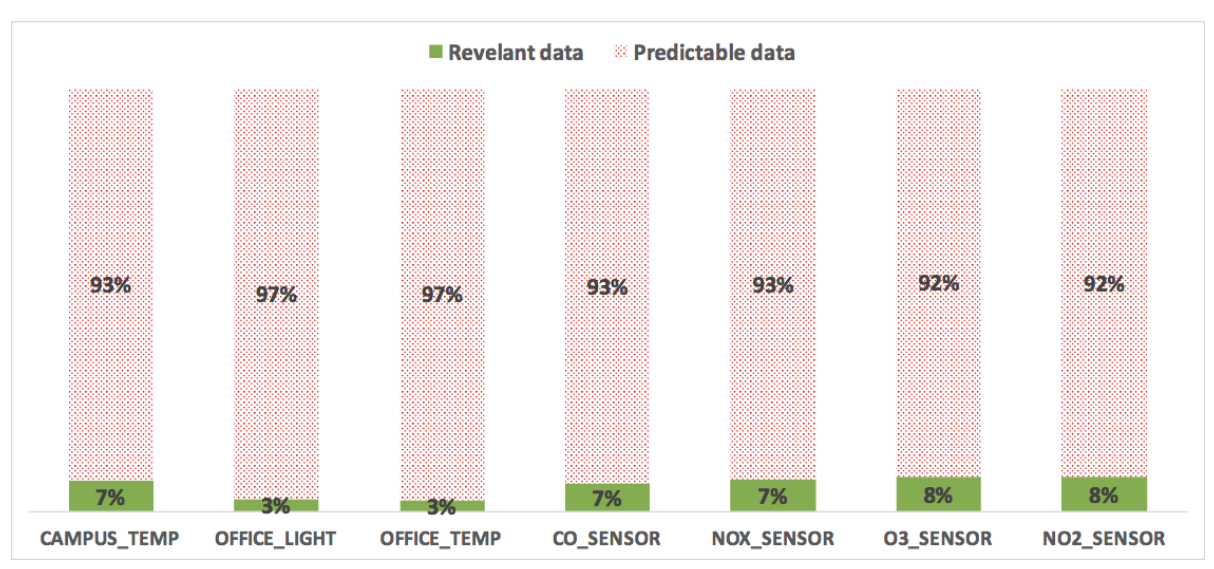

Figure 3: Predictable data from real-environment datasets

values are collected from periodic sensors deployed in an Italian city [15].

The lifetime of batteries powering devices deployed in sensor networks can be extended significantly when sensors are running in a deep-sleep mode. During a deep-sleep period, a sensor does not sample or send data over the network. Machine learning algorithms learn from historical data to predict, within a margin of error, a current or future values without requiring any call to the sensor network. In addition to prediction capability, live learning analysis are suitable to reduce the number of values transferred through the network. One of these methods uses polynomial segmentation [17] to represent a phenomenon using a sequence of polynomials functions. In a nutshell, this approach fit a polynomial function while data are received from sensors and append a new polynomial function when a significant change is observed. Polynomial functions are later able to extrapolate any points between the first and last inserted one. The application of this algorithm on the datasets presented in TAB. 1 shows that more than $90 \%$ of the values can be extrapolated from the polynomial functions, $c f$. FIG. 3, as they fit the previously learn change trend. Considering that these data can be extrapolated, from both sensor and collector server sides, their mea- 


\begin{tabular}{|c|c|c|c|c|}
\hline WSN layer & Network layer & Related work & Our approach & \\
\hline $\begin{array}{l}\frac{\overline{0}}{\bar{ष}} \\
\frac{.0}{\frac{0}{2}} \\
\frac{0}{2}\end{array}$ & Data acquisition & [20] & 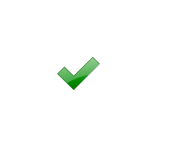 & ర్ర \\
\hline \multirow{3}{*}{$\begin{array}{l}\text { 辛 } \\
\text { 总 } \\
\text { ż }\end{array}$} & Transport & \multirow{2}{*}{$\begin{array}{c}\text { [17] [18] } \\
{[19]}\end{array}$} & & $\frac{1}{3}$ \\
\hline & Routing & & & ڤ్ \\
\hline & MAC Protocol & [16] & $\vartheta$ & \multirow{3}{*}{$\frac{\overline{0}}{\overline{0}}$} \\
\hline \multirow{2}{*}{ 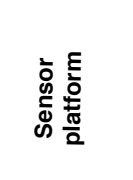 } & Duty cycle & [14] [15] & $\gamma$ & \\
\hline & Hardware & [11] [12] [13] & & \\
\hline
\end{tabular}

Figure 4: Cross-layer power management: positioning of your approach

surement and transfer over the network can be saved. For instance, over 15 days, 3823 values have been collected from a temperature sensor (CAMPUS_TEMP). This corresponds to 255 measurements and transfers per day. The application of the polynomial segmentation saves $93 \%$ of the values, which shows that only 18 measurements and transfers per day are sufficient to characterize the phenomenon.

\subsection{Impact of automatic buffers configuration based on sensor data use fre- quency}

Power management is a dominant issue in sensor networks as sensor nodes have a limited amount of energy that determines their lifetime [18]. Energy optimizations are performed at each layer of the protocol stack used by sensing infrastructures ( $c f$. FIG. 4). At the sensor platform stage, power management optimizations mainly target hardware and duty cycle, i.e., the fraction of one period in which the system is active, concerns. At the hardware layer, optimizations target mainly battery [19, 20] and platform electronic design [21]. For example, although the platforms ESP-12E and ESP-12 DevKit are built around the same chip, there is a 4.6 ratio between their respective energy consumption: $70 \mathrm{~mA}$ for ESP-12E DevKit and $15 \mathrm{~mA}$ for ESP-12E in stand-by mode. These consumptions fall respectively to $200 \mu A$ and $10 \mu A$ in deep-sleep mode. At the duty-cycle level, optimizations are mostly made by dynamic context-adaptation [22, 23]. With such an adaptation, it becomes possible to set automatically a platform in a deep-sleep mode whenever it is not used for a significant time. At the network level, power management optimizations are 
often made in medium access protocols (MAC), routing protocols and transport protocols [24]. At the routing and transport layers, we can observe that many energy efficient protocols have been proposed for WSNs [25, 26, 27]. At the application level, energy optimizations address mainly the data acquisition operations.

These optimizations are tedious to create as applications are outside the WSNs and designed by software engineers that might not have a fully understanding of the underlying sensing infrastructures. Applications are likely to over-exploit the sensing infrastructure by acquiring data at a tremendous period, thus draining batteries to ensure an optimal quality of service. The IETF ${ }^{6}$ Protocol Suit for the Internet of Things proposes a request/response interaction using CoAP [28]. CoAP offers proxy and caching mechanisms allowing last produced values to be acquired without sending requests within the sensor network. Another solution consists in the introduction of a data buffer at the sensor platform layer. Data stored in the buffer are then only sent when needed by applications. Such a method can massively reduce the energy consumption. For instance, according to the ESP 8266 datasheet [11], the microcontroller consumption varies between 120 and $170 \mathrm{~mA}$ during data transmission which is ten times higher than the power consumption when the network interface is off.

\subsection{Towards an adaptive approach for saving energy}

In the previous subsections, we have made the following observations :

- $\left(O_{1}\right)$ many physical phenomena have a predicable nature [29]. Therefore we can take advantage of this characteristic to dynamically adjust the sampling period of sensors;

- $\left(\mathrm{O}_{2}\right)$ applications leveraging sensor data could query them at a frequency lower than the sampling rate needed to capture properly the physical phenomenon. Therefore, we can take advantage of the data usage to adjust the data sending period.

We propose to combine these two observations to opportunistically optimize sampling periods where sensors can configure themselves in deep-sleep mode $\left(c f . O_{1}\right)$ and optimize sending periods to send data only when they are relevant to applications $\left(c f . \mathrm{O}_{2}\right.$ ). We expect the combination of these periods to enhance the battery life-time. Thus, we address the following research question: $(R Q)$ "Can we significantly gain battery lifetime for a whole sensor platform using adaptive sampling and sending periods?"

To tackle this energy optimization problem, we present in the following a tooled approach working at duty cycle, MAC protocol and data acquisition layers, $c f$. Fig. 4. This tooled approach relies on machine learning techniques that adapt dynamically (i) the sampling period according the predictability of data (Data acquisition and Duty cycle layers) and (ii) the sending period according

\footnotetext{
${ }^{6}$ Internet Engineering Task Force
} 
the average use by the applications (Data acquisition and MAC protocol layers). Regarding the routing and transport layers, we rely on classical IP routing and UDP protocol.

We conducted an experimental evaluation of the proposed approach on real battery-powered platforms to measure the gain in battery lifetime brought by adaptive periods. For this purpose, the platforms were equipped with a temperature sensor and were deployed in a environment where we already had datasets describing the past temperature values. Thus, the computed sampling periods were expected to be related to the temperature variation of the environment. We also mocked several application collective behavior to obtain relevant computed sending periods based on the data usage.

\section{Predictive Model}

In the previous section, we have discussed how a sensor platform battery is directly impacted by sampling operations, i.e., acquisition of a value, and the network access, i.e., the sending or the retrieval of information through the network. Machine learning techniques can help to predict futures values and thus, reduce the amount of sampling operations. We have also seen that some applications could not require, in a real-time manner, the values measured by sensor platforms. We can also use machine learning to predict when data are necessary and thus, reduce the amount of sending operations.

In this section, we address the adaptive aspect of the research question by introducing a predictive model to compute the appropriate collection periods, i.e., the appropriate sampling and sending periods.

\subsection{Assumptions on the sensing infrastructure}

We assume a sensing infrastructure built on three layers (cf. Fig. 5): (i) a sensor network layer containing the sensor platforms that host the sensor and measure environmental phenomena, (ii) a middle layer containing a middleware that stores the sensors values and the sensors configuration, and (iii) an application layer containing third party applications that are fed with sensor values. The sensors send their values to the middleware and retrieve their configuration from this latter.

We also made the following assumptions on the sensor platforms:

- Sensor platforms are powered by batteries and their replacement is a tedious maintenance operation;

- sensors have a deep-sleep mode allowing them to save energy efficiently when they are not on duty;

- sensors have a writable memory to store their configuration and to buffer their measurements (deep-sleep modes do not guarantee a full retention of RAM) ; 


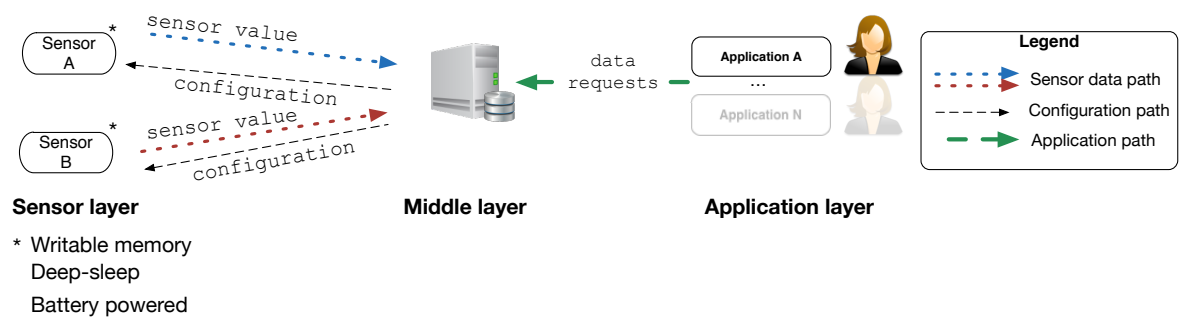

Figure 5: Overall sensing infrastructure considered in this paper

- sensors platforms are directly connected to an IP network and can retrieve a new configuration upon demand;

- sensors platforms have limited computation facilities as they are often built on microcontrollers.

\subsection{Building a predictive model}

We propose to build a predictive model to extrapolate, for a each sensor, (i) its future values based on its past values and (ii) user's requests from its activity history in order to reduce the amount of sampling and sending operations.

However, retaining all sensor past values hardly scales in terms of storage volume. In statistics, linear regression allows one to interpolate (within an acceptable margin of error) a polynomial function from a set of values. Thus, the resulting polynomial function contains in itself the set of values. We leverage this technique on sensor values to store only polynomial functions (describing a set of sensor values) rather than storing raw sensor values. The same observation can be made over activity history. Instead of storing every single sensor's activity, we compute the overall activity of the sensor, per time-slot.

Given that the computation of these interpolations is resource-intensive, we rely on a middleware located outside the sensor network where servers have higher computing and storage resources suitable for big data analysis. Our predictive model is built upon the GreyCat ${ }^{7}$ graph database [17] that integrates, directly into nodes, machine learning algorithms.

We represent every sensor deployed in the sensor network in a model containing a sensor node collecting the received values, a compressed node, i.e., a node performing the autoregression operations, and an activity node, i.e., a node storing the sensor's activity ( $c f$. FIG. 6).

\footnotetext{
${ }^{7}$ https://github.com/datathings/greycat
} 


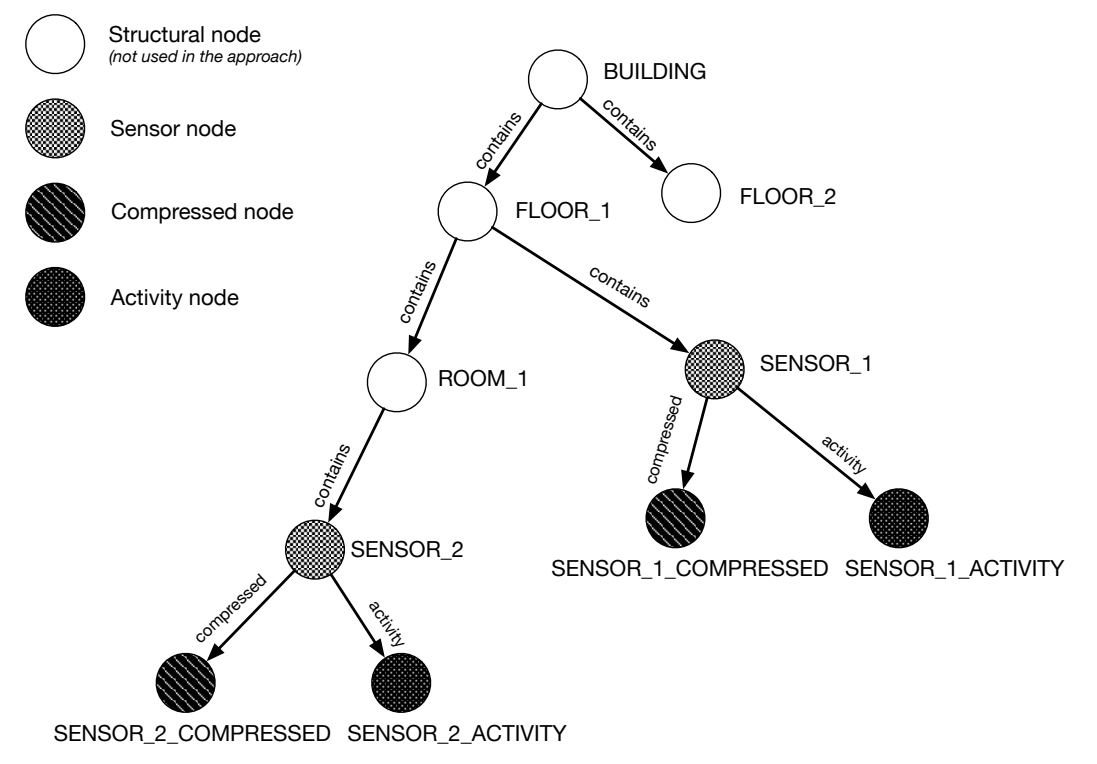

Figure 6: Excerpt of a Smart Building representation with activity and compressed nodes for each sensor

Sensor node. A sensor node is responsible for collecting data retrieved from its associated sensor in the network. This node has a value attribute updated with the latest value coming from the represented sensor. Historical values can be retrieved by reverting the node at a previous time. In the case no value has been stored at the requested time, the node retrieves automatically the freshest previous value.

Compressed node. A compressed node stores sensor data using a polynomial live segmentation. Polynomial live segmentation is a technique that builds and updates a polynomial function modeling the current trend of data, as shown in SEC. 2. This function is kept as long as it 'fits' newly measured values, when the contrary happens, an empty polynomial function is created and chained with the previous one.

The complete process of live polynomial segmentation is depicted in Figure Fig. 7.

The chaining mechanism works as follows: when a new timestamped $t$ value needs to be inserted, the algorithm gets the closest segment to $t$. If no segment is found, a new polynomial $f_{\text {new }}$ (such that $\operatorname{deg}\left(f_{\text {new }}\right)=0$ and $f_{\text {new }}=v$ ) is created. Otherwise, the algorithm checks if the value fits the polynomial $f$ closest segment within a certain precision (chosen by a domain expert). As long as the value fits the closet segment, the polynomial does not need to be updated. Conversely, the algorithm tries to increase $f$ 's degree and re-train $f$. 


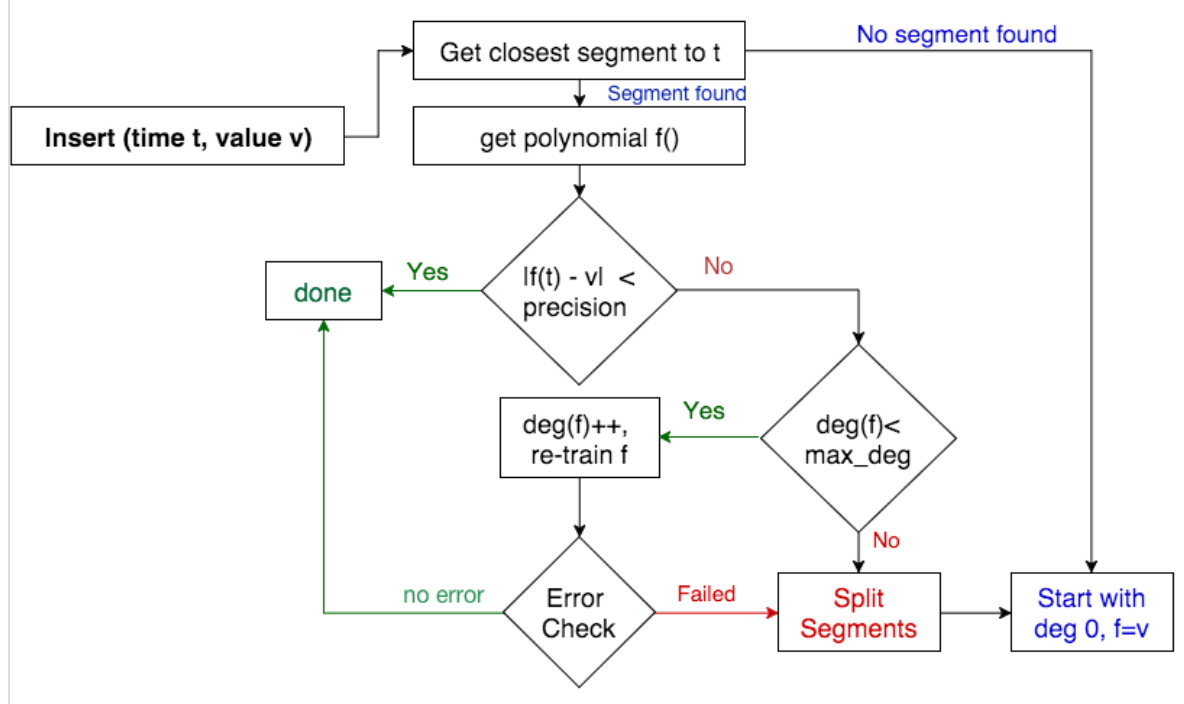

Figure 7: Polynomial live segmentation algorithm

In the event $f$ cannot be updated, the algorithm performs a segmentation and builds a new polynomial $f_{\text {new }}$.

In other words, whenever data coming from the sensor network is collected, the polynomial live segmentation checks whether it suits the current polynomial function or stays within an acceptable margin of error, i.e., no significant change, and then will either increase its degree or leave it unchanged. If the data indicates a significant change, a new polynomial function, continuous to the previous one, will be built.

As a result, the compressed node contains only polynomial functions to represent the data. The ratio between the number of polynomial functions and the number of values stored in the sensor node gives the compression rate.

In [17], the authors of the live polynomial segmentation algorithm gave a mathematical evidence about the ability of this chain of polynomial functions to rebuild the original measured values within a given tolerated error. They also already pinpointed the relation between signal steady state and unchanged polynomial degree after insert. Besides the compression of such segmentation algorithm, this steady state detection is later reused in this paper to compute the optimal sleeping time of sensors.

An illustration of this live segmentation algorithm against a simulated signal is shown on FIG. 8. The polynomial reconstruction (black line) interpolates the original data (red crosses) with a certain precision. As long as the original data fits the polynomial or the current polynomial's degree can be increased, no segmentation is required. Conversely, when the original data's trend varies greatly (e.g., switching from a variable trend to a steady trend, as shown between 


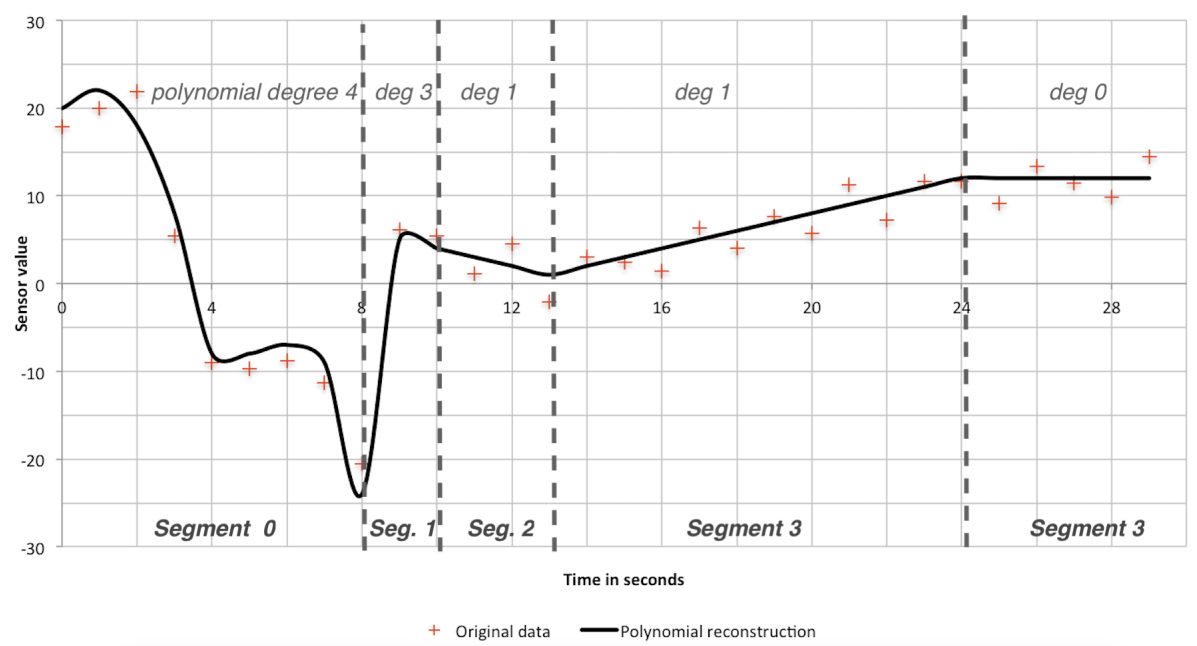

Figure 8: Illustration of the polynomial live segmentation algorithm

Access to temperature measures (\#slots $=24$, May 2016, UTC time)

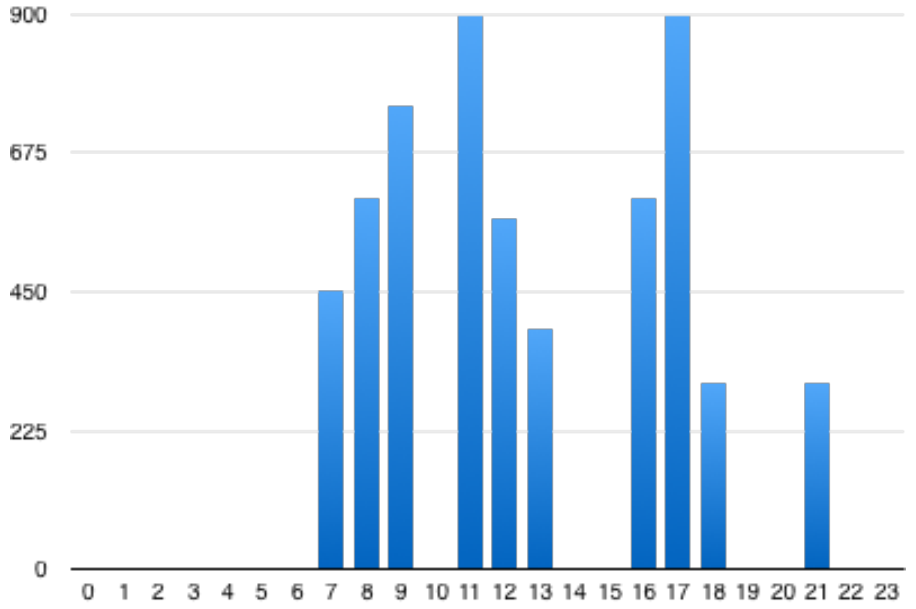

Figure 9: Cumulative daily temperature measures accesses (one month)

segments 1 and 2), the algorithm can no longer update the polynomial and builds a new one starting at degree 0 in a new segment.

Activity node. An activity node logs the statistics about data requests using a gaussian slots representation. These Gaussian slots allow us to predict, given a time slot, the frequency of requests by third-party applications. They are up- 


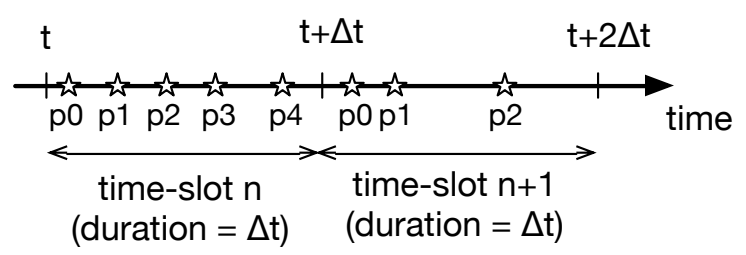

Figure 10: Polynomial functions distribution across time

dated in live, i.e., every time an application requests a data. For instance, the figure FIG. 9 has been obtained from the logging of temperature data accesses during one month and the distribution of these logs in gaussian slots shows that 900 accesses to the temperature produced between $5 \mathrm{pm}$ and $6 \mathrm{pm}$ UTC have been logged in time slot \#17. Consequently, these values are highly requested and thus the sampling period needs to be shortened to ensure the data availability. Conversely, values produced at night (e.g., 0-6am UTC: time slots $\# 0-6)$ are never requested by the application and thus, the sending period can be increased to save energy. In the following, we define the activity of a sensor as the number of data accesses during a time slot.

\subsection{Optimal data sampling period}

As stated previously, it is irrelevant to sample data if the observed phenomena can be predicted, e.g., a temperature room remains steady. We thus propose to adapt the sampling period according to the number of significant changes (i.e. the amount of different polynomial functions) in a time-slot. Indeed, a change of polynomial function happens every time the measured value cannot fit further the current prediction. We introduce $T$ the set of time-slots and $\Delta t$ the duration of a time-slot. We also define $P_{t}=\left\{p_{0}, p_{1}, \ldots p_{n}\right\}$ the set of polynomial functions in a given time-slot $t \in T$ and time $(p)$ the time associated to the beginning of a polynomial function (FIG. 10). As it is irrelevant to perform sample operations while the prediction remains valid, we compute, for a time-slot $t \in T$, the time between the beginning of two consecutive polynomial functions, i.e., the amount of time the prediction remains valid:

$$
\Delta p_{t}=\left\{\operatorname{time}\left(p_{n+1}\right)-\operatorname{time}\left(p_{n}\right)\right\}, 0 \leq n<\operatorname{card}\left(P_{t}\right)
$$

If a time-slot $t \in T$ contains only one polynomial function $p_{0}$, we compute the maximum time between time $\left(p_{0}\right)$ and the time-slot endpoints, such as $\Delta p_{t}=\max \left(\Delta t-\operatorname{time}\left(p_{0}\right)\right.$, time $\left.\left(p_{0}\right)\right)$. In the case the time-slot $t \in T$ is empty and in order to have at least one measurement per time-slot, we define $\Delta p_{t}=\Delta t$. These two latter computations can allow the detection of abnormal situations (e.g., a change of temperature because of the sudden heating of a room) in order to integrate these abnormal values into the computation of future polynomial functions. For each time-slot, we compute the minimal time 
between two polynomial functions to ensure the best data quality. Thus, we define the set of minimal sampling periods Psamp $_{t \in T}$ as follows:

$$
\operatorname{Psamp}_{t}=\min \left(\Delta p_{t}\right)
$$

Then, the data sampled is stored into a buffer located in a non-volatile memory (e.g., an $\mathrm{EEPROM}^{8}$ ) and the platform enables the deep-sleep mode until the next data sampling or data sending.

\subsection{Optimal data sending period}

As shown on FIG. 9, data are not always used in real-time or critical applications and therefore, their sending can be deferred to save energy. Thus, we propose to configure the sending period according to the expected use of the data and to buffer the data between two sendings. We reuse the same set of time-slots $T$ and $\Delta t$ the duration of a time-slot as introduced before. Given an activity $a(t)$ produced in time-slot $t \in T$ and $A_{t \in T}$ the set of all activities produced in $t$, we define the set of minimal sending periods $P s e n d_{t \in T}$ as follows:

$$
\operatorname{Psend}_{t \in T}=\Delta t / \operatorname{card}\left(A_{t}\right)
$$

The obtained $P \operatorname{send} d_{t \in T}$ value corresponds to a fair distribution over time of the activities. For example, if four activities has been recorded within a time slot and $\Delta t=60 \mathrm{~min}$, the value Psend would be equal to $60 / 4=15 \mathrm{~min}$. Thus, the sensor platform would send its buffered values every fifteen minutes.

\subsection{Adaptive firmware}

Sensor platforms need to retrieve the optimal sampling and sending periods computed on the server-side. To do so, we propose an algorithm that measures data, buffers them and periodically connects to the remote server to send data and retrieve the appropriate sending and sampling periods. The algorithm is depicted in Alg. 1 and can be implemented on platforms with writable memory and connecting facilities, e.g., ESP8266 or RTL8710 based-platforms.

First of all, the sensor platform retrieves the current timestamp (line 1) and the timestamps matching the next sampling and the next data sending (lines 2-3). If the next sampling time is outdated (line 4), the platform performs a measurement that is buffered thereafter. Finally, the platform reads from memory the current sampling period and updates its next sampling time. If the next sending time is outdated (line 10), the platform prepares itself for sending the buffered data over the network. After having sent and flushed the data buffer, the platform retrieves its updated sampling and sending periods. It also updates its current timestamp using the NTP protocol. In a final step, the platform puts itself in deep-sleep until the next sampling or sending (line 26). 


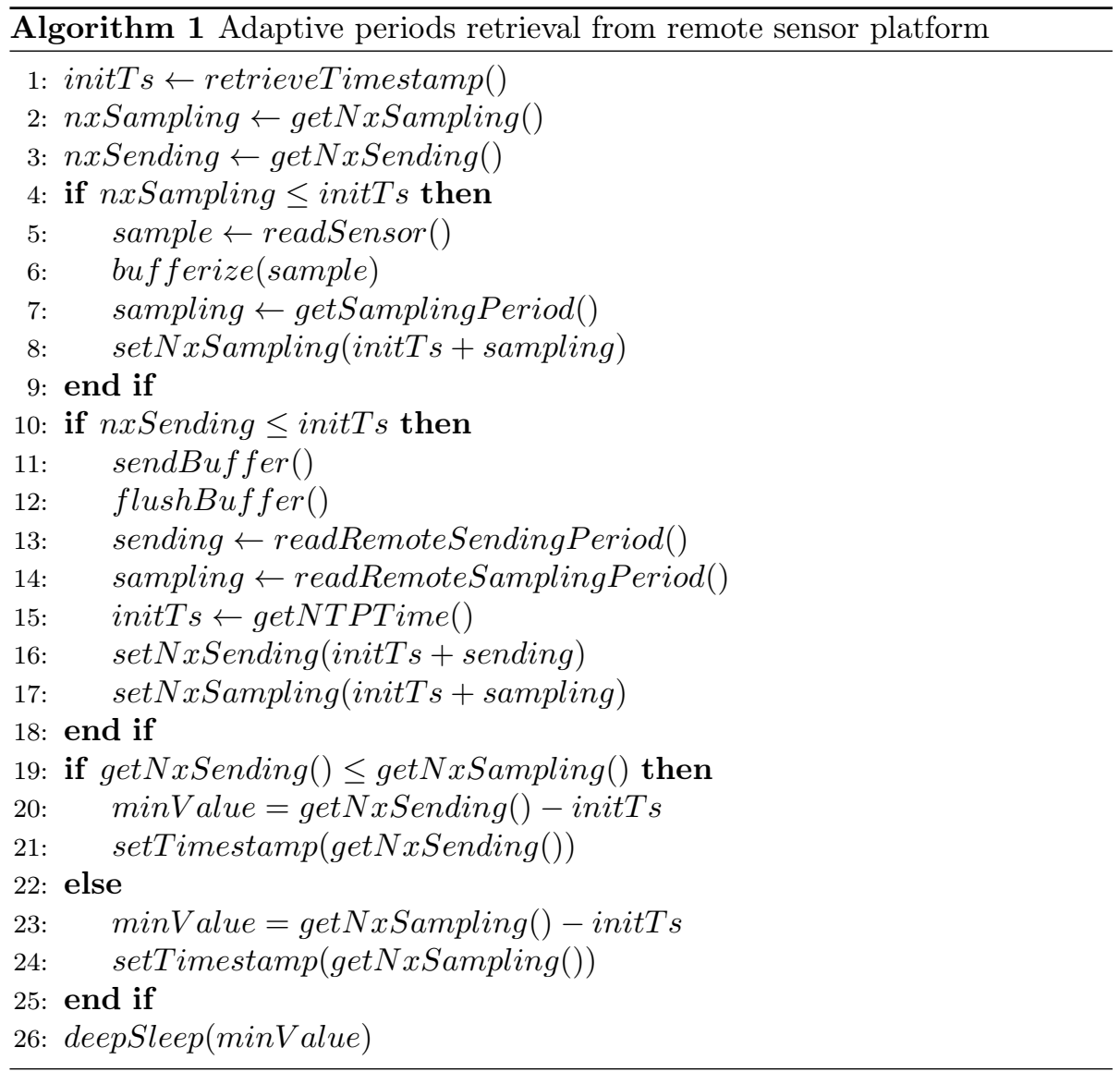

\subsection{Resulting architecture}

From the contributions presented in this section, we depict on FIG. 11 the implementation of the reference architecture presented in FIG. 5.

Sensor platforms layer. This layer contains all the sensor platforms targeted by our approach. They execute an implementation of the adaptive firmware presented in ALg. 1. A server located in the middle layer is responsible to collect the sensor data and to compute the appropriate sampling and sending periods.

Middle layer. A server located in this middle layer is responsible for maintaining the data structure presented in Fig. 6. Each time a sensor value is received from a sensor platform, the middle layer updates its corresponding nodes with the freshest value and foresees the next data sampling and sending periods. In

${ }^{8}$ Electrically Erasable Programmable Read-Only Memory 
addition, for each periodic node, the middle layer updates the activity node each time a third-party application performs a sensor value request.

Applications layer. This layer contains the set of third-party applications that retrieve sensor values.

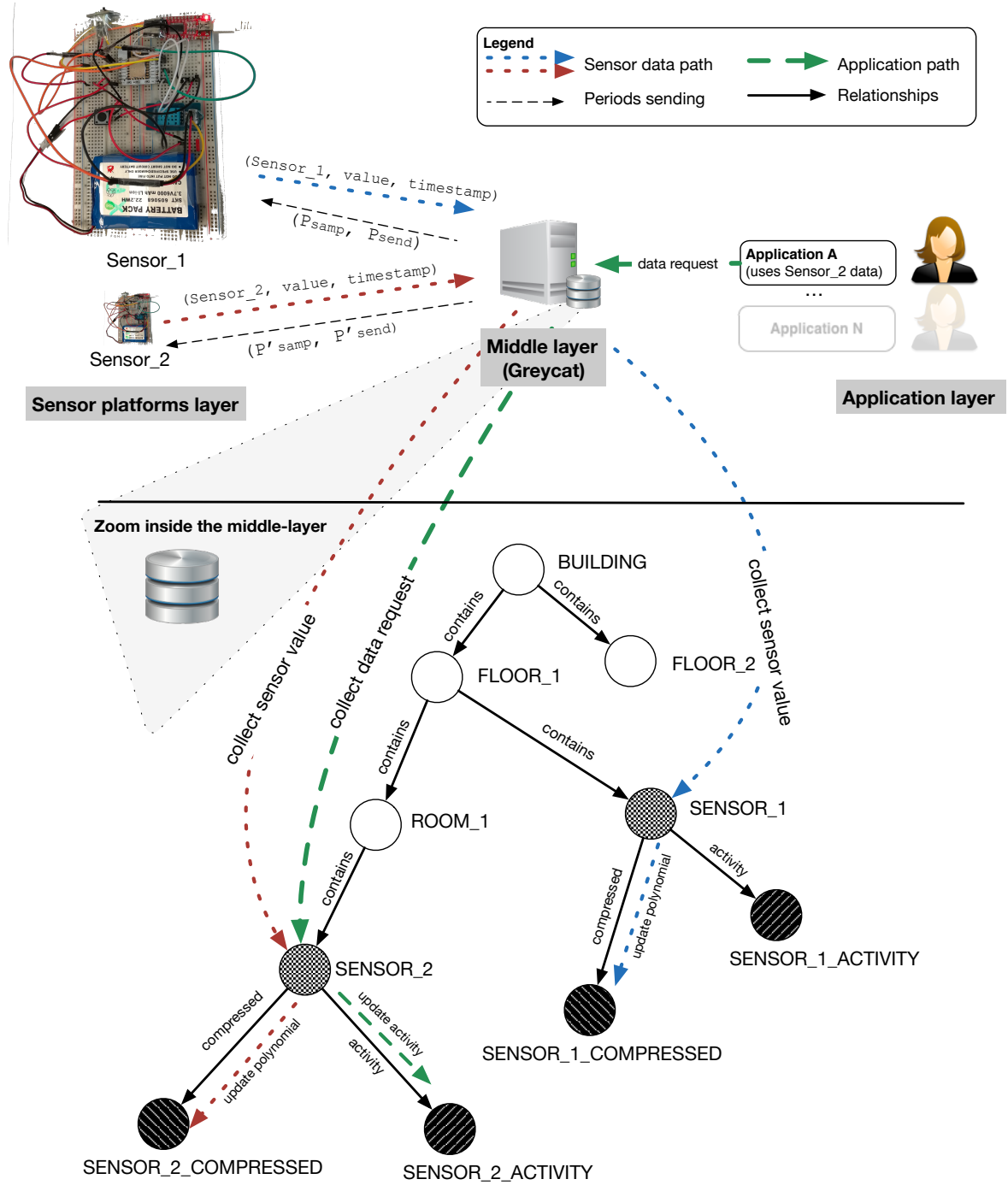

Figure 11: Reference architecture for computing and sending adapted periods 


\section{Measuring the gain in battery lifetime}

In the previous section, we have introduced a predictive model allowing the computation of adaptive sending and sampling periods based on the predicted values of the considered physical phenomena and on the predicted use of data by third-party applications. We focus in this section on measuring the gain in battery lifetime brought by this model (i.e., measuring aspect of our research question). We perform an experimental assessment on a prototypical sensor platform based on an ESP8266 microcontroller by conducting three experiments:

- (Exp.1) aims at measuring the battery life-time of the sensor platform sampling and sending values at fixed periods without deep-sleep. The voltage at the moment the platform ceases to work will be considered as the cut-off voltage, i.e., the minimum voltage required by the platform to work properly. The following experiments will measure the time required to reach this value from a charged battery;

- (Exp.2) aims at measuring the time required to reach the cut-off value of the sensor platform sampling and sending values at fixed periods using deep-sleep. These values will be considered as reference values to measure the gain brought by our approach;

- (Exp.3) aims to empirically show that our approach increase the battery lifetime. Instead of fixing the periods, we use our contribution to dynamically compute adaptive sampling and sending periods.

\subsection{Experimental setup}

To perform the evaluation of the approach, we have instantiated the reference architecture ( $c f$. FIG. 11) with the following elements:

Sensor platform layer. It contains platforms relying on an ESP8266 microcontroller and on a temperature sensor. This microcontroller is largely used by the IoT industry and offers an efficient deep-sleep mode, a Wi-Fi connectivity and a writable EEPROM that can be used to buffer data. In order to avoid any unwanted energy leaks, we have built an electronic design using the strictly necessary passive components. All the sensor platforms rely on a $3.7 \mathrm{~V} 6000 \mathrm{mAh}$ lithium-ion battery.

Middle layer. It relies on a middleware ${ }^{9}$ developed using Java 7 and Scala 2.11 languages and using the GreyCat technology. Greycat allows the middleware to trigger in a reactive way machine learning executions using the concepts presented in SEC. 2. The middleware builds automatically the prediction model presented in SEC. 4 and updates the nodes (cf. Fig. 6) each time a sensor value is collected.

\footnotetext{
${ }^{9}$ The source code is available on Github: https://github.com/ulrich06/greycat_senso
} 
Table 2: Initial settings for the 3 experimental contexts

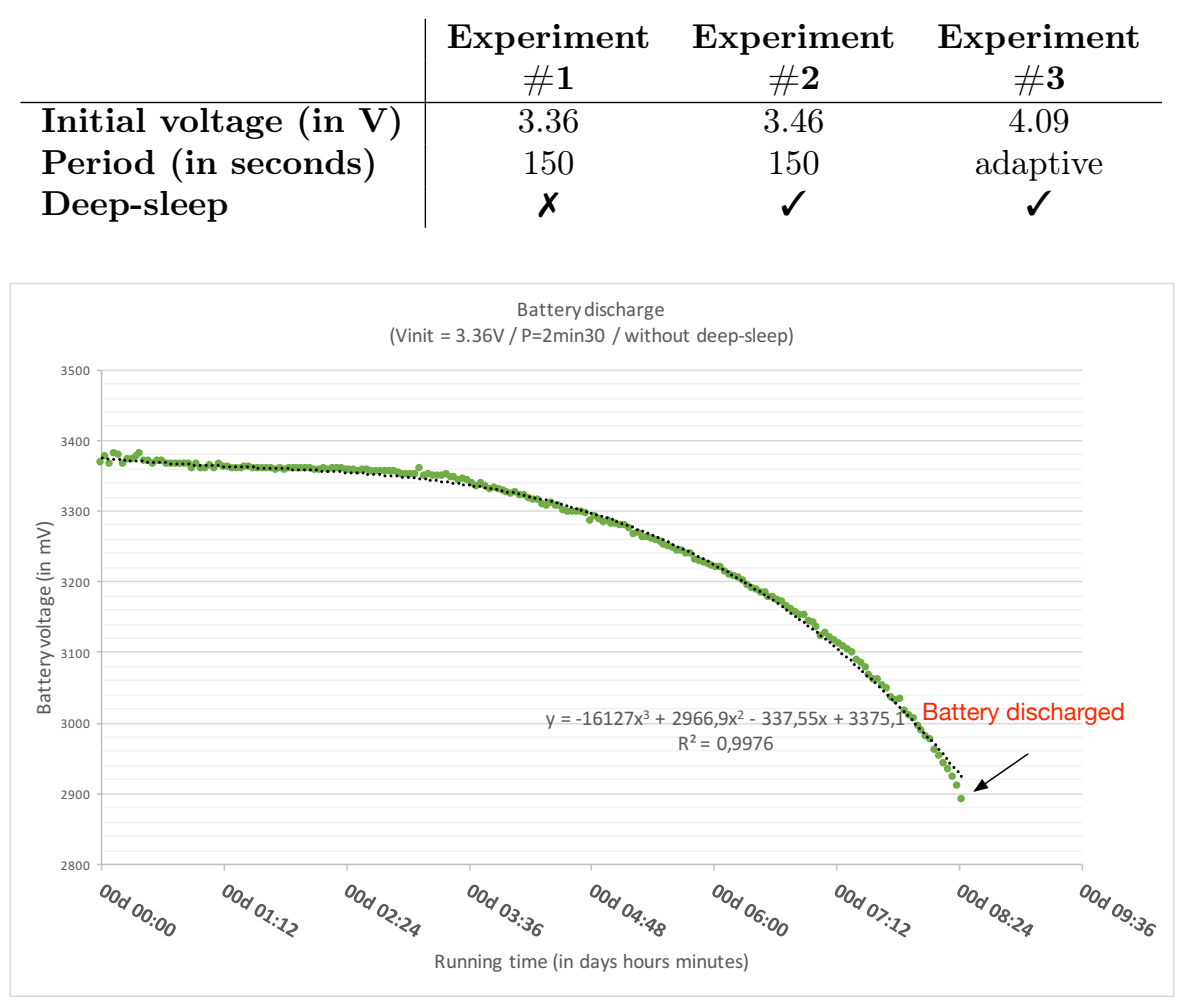

Figure 12: Battery discharge without deep-sleep (Exp.1)

Application layer. On this side, we have mocked an application behavior using the collected temperature values.

For each experiment, we use a charged Li-ion battery and preconfigure, if necessary, the sampling/sending periods and the deep-sleep mode ( $c f$. TAB. 2).

\subsection{Standard battery discharge (Exp.1)}

This first experiment aims to illustrate the lifetime of a battery-powered platform that does not use deep-sleep facilities nor adaptive periods. In order to conduct a time-limited experiment, we overexploit the platform by sampling and sending temperature values associated with the current voltage value at the battery terminals every 150 seconds. This value allows 24 measurments per hour simulating, at a speed increased by 24 , a sensor performing one measurment per hour. Moreover, the undersampling of the voltage measurements mitigates the invasive effect of the measure, which also affect the battery.

The obtained voltage values are plotted on Fig. 12. We can observe that the voltage values decrease following a cubic function. The last value obtained 
$(\sim 3.0 \mathrm{~V})$ corresponds to the cut-off value mentioned by the constructor [11] (i.e., the minimal voltage value for operating the platform) and has been reached after $T_{\text {cut }}=8.5 \mathrm{~h}$. By extrapolating this result towards a platform that performs only a single sampling and sending operation per hour (minimum requested, $c f$. hypothesis), i.e., the platform is used at a rate divided per 24 , the battery lifetime is then increased by 24 and reaches $T_{c u t}=8.5$ days .

In our context (performing at least one measurement per hour), this shows that deploying such a battery-powered sensor platform without using deep-sleep facilities will lead to important maintenance operations as the battery will have to be replaced on a weekly-basis.

\subsection{Battery discharge with deep-sleep (Exp.2)}

The ESP8266 platform offers a deep-sleep facility that lowers the power consumption down to $10 \mu A$ [11]. This second experiment aims at comparing the newly discharge curve with the one obtained in the first experiment in order to show how deep-sleep increase the platform lifetime. It also aims at showing whether the deep-sleep periods are sufficiently accurate regarding the periods desired by the user. For this experiment, we kept the same $P=150$ seconds sampling/sending period as defined in Exp.1 and we enabled the deep-sleep facilities between two cycles.

The obtained voltage values are plotted on FIG. 13. To limit the experiment in time (an ESP8266 theoretically can stand for months using the deep-sleep facilities), we stopped the experiment before reaching the cut-off value. Despite some measurements artifacts (due to physicochemical properties of the battery - these artifacts, due to their low absolute value, are not representative), we observe that the curve follows a linear decrease $(0.1 \mathrm{~V}$ are lost every 2 days). From an initial voltage value of $3.46 \mathrm{~V}$, the extrapolation of the curve depicted in FIG. 13 shows that the cut-off value $(3.0 \mathrm{~V})$ is reached in 8 days. The application of the deep-sleep facilities to a platform performing only a single sampling and sending operation per hour can scale the battery lifetime up to $8 * 24=192$ days.

Through this experiment, we also wanted to check if the platform was correctly inactive during deep-sleep periods, i.e., no sampling and sending operations are performed and if the requested sleeping periods were correctly executed. We have thus extended the implementation of the algorithm presented in ALG. 1 to $\log$ on the serial port all the operations performed on the platform. Then, we have connected an oscilloscope to the output of the serial port and plotted the values obtained on the figure Fig. 14. The signal analysis shows a platform stays woken-up $\left(T_{w 1}, T_{w 2}, T_{w 3}\right) 9$ seconds on average (the connection time to the wifi network is not deterministic and can therefore influence these values) and sleeps $\left(T_{s 1}, T_{s 2}\right) 145$ seconds on average. Thus, an ESP8266 platform is active only $6 \%$ of a wake-up/sleep cycle. In particular, we notice that, as expected, no operations are performed during the deep-sleep. We can also notice that the periods $P_{1}$ and $P_{2}$ are close to the wished period of 150 seconds. This inaccuracy is caused by the lack of precision of the micro-controller clock. 


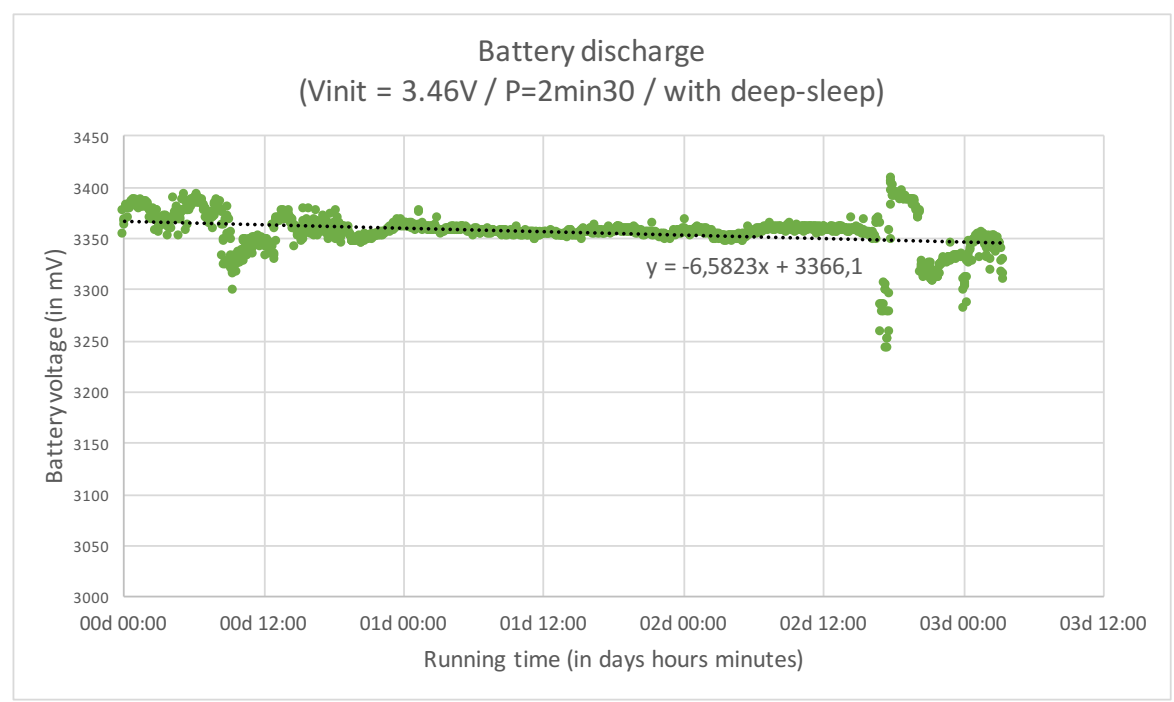

Figure 13: Battery discharge with deep-sleep (Exp.2)

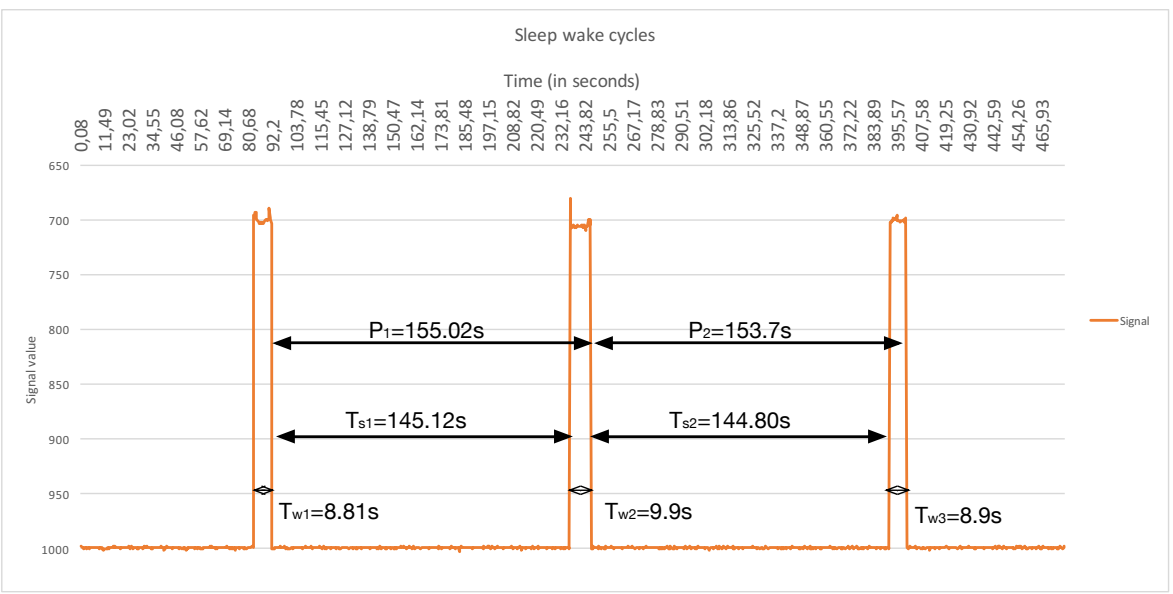

Figure 14: Wake-up/Sleep cycles (Exp.2)

Nevertheless, despite this slight imprecision, we can consider that the use of the deep-sleep respects the periods wished by the user.

\subsection{Dynamic approach (Exp.3)}

In this experiment, we propose to empirically show that adaptive periods contribute to increase the battery lifetime. We have implemented the firmware 


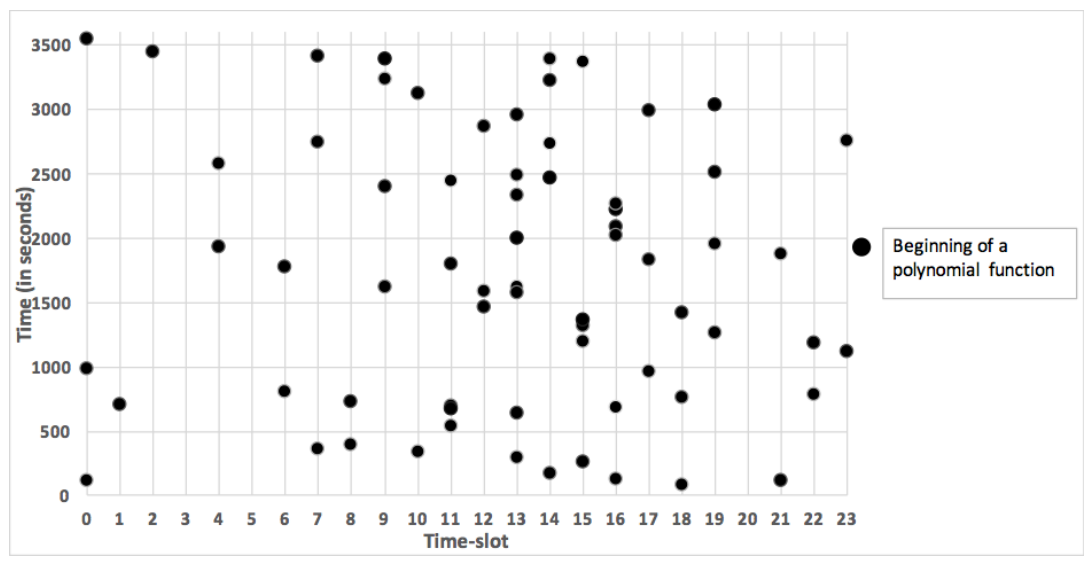

Figure 15: Polynomials distribution. (Exp.3)

A dot represents the beginning time of a polynomial in its time-slot

presented in Alg. $1^{10}$ and we have deployed it on our prototypical sensor platform, as illustrated on the left hand-side of FIG. 11.

Set up. For this experiment, we set the following parameters :

- $\Delta t=60$ minutes in order to have at least one temperature measurement per hour.

- $\operatorname{card}(T)=24$ in order to reason over a day.

- Precision of the prediction: 1 celsius degree to match the temperature sensor accuracy (which we consider as accurate enough for an office).

\subsubsection{Sampling period}

The computation of the appropriate sampling periods is performed accordingly to the polynomial functions computed by the compressed node To obtain initial polynomial functions, we fed the sensor node ( $c f$. SENSOR on FIG. 6) with a 15 days dataset containing 3210 values and describing the temperature in the office under experiment. We configured the compressed node to accept 1.0 celsius degree as an acceptable margin of error because of the accuracy of the temperature sensor.

The polynomial regression performed by the compressed node results in 66 polynomials describing the learned data with a precision of 1.0 celsius degree. The figure Fig. 15 shows the number of polynomial function per time slot and their distribution within the time slot. The greater the number of polynomials in a given time slot, the more the physical phenomenon has changed and therefore

\footnotetext{
${ }^{10}$ https://github.com/ulrich06/Senso
} 


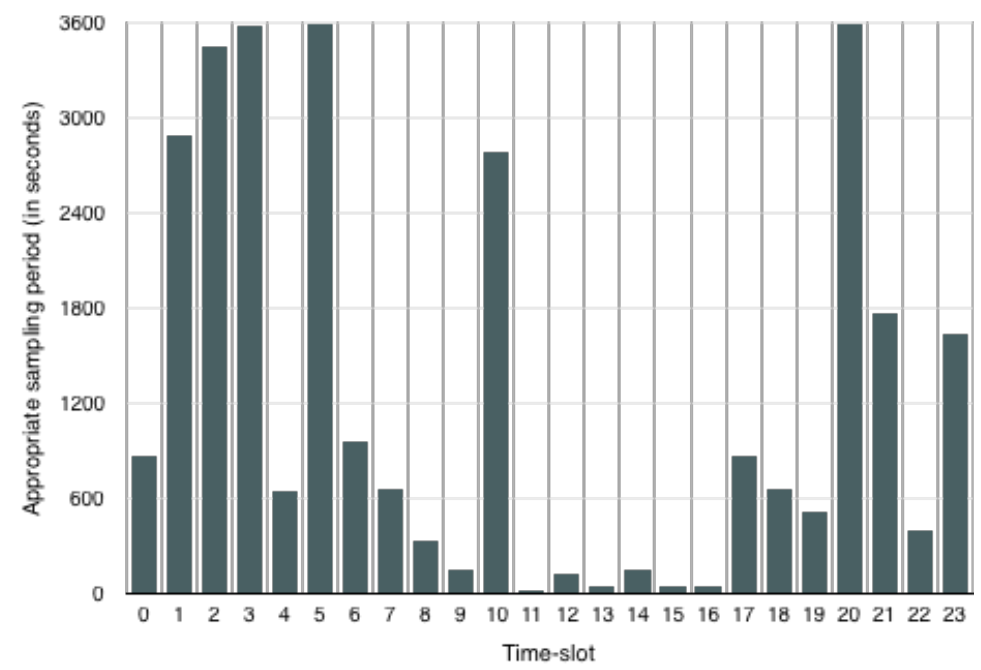

Figure 16: Computed daily sampling periods. During time-slot \#10, the door is often closed, i.e., the temperature remains stable (Exp.3)

the sampling period must be reduced accordingly. According to this figure, we can observe that the most frequent changes occur during time slot \#13. The more two beginnings of polynomials are separated in time, the more slowly the physical phenomenon varies. Thus, it is relevant to use the prediction result rather than to make an energy-intensive measurement. According to this figure, during time slot $\# 0$, we observe that the temperature varies slowly and, during time slot $\# 16$, the temperature fluctuates quickly. We report on FIG. 16, the result of the sampling period computation from the application of EQ. $2^{11}$ over the $\Delta p_{t}$ values. When large temperature variations are expected to occur, the sampling periods are decreased to ensure that the most data are sampled. Conversely, when the temperature is expected to remain stable, the sampling periods are increased to save the battery life-time.

In TAB. 3, we apply the polynomial segmentation on the datasets presented in TAB. 1 and extrapolate what would have been the autonomy of an ESP8266 platform considering a duty cycle of 9 seconds ( $c f$. FIG. 14), an average operating current of $80 \mathrm{~mA} \mathrm{[11]} \mathrm{and} \mathrm{a} 6000 \mathrm{mAh} 3.7 \mathrm{~V}$ battery. Thanks to the polynomial segmentation algorithm, the number of values required to characterize a signal is greatly reduced (we showed in SEC. 3.1 that less than $10 \%$ of the values can describe the whole dataset). This reduction in the number of values leads to greater deep-sleep periods (minimal operating current, ca. 20 $\mu A$ ) and thus contributes to increasing the battery lifetime. By calculating the duty

\footnotetext{
${ }^{11}$ computing the set of minimal sampling periods $P s a m p_{t \in T}$
} 
Table 3: Theoretical gain in battery lifetime after using polynomial segmentation

\begin{tabular}{|c|c|c|c|c|}
\hline $\begin{array}{c}\text { Sensor } \\
\text { (compression) }\end{array}$ & $\begin{array}{c}\text { Duty cycle } \\
\text { before comp. } \\
\text { (s) }\end{array}$ & $\begin{array}{l}\text { Duty cycle } \\
\text { after comp. } \\
\text { (s) }\end{array}$ & $\begin{array}{c}\text { Estimated } \\
\text { Battery } \\
\text { lifetime } \\
\text { (before } \\
\text { comp., } \\
\text { in days) }\end{array}$ & $\begin{array}{c}\text { Estimated } \\
\text { Battery } \\
\text { lifetime } \\
\text { (after } \\
\text { comp. } \\
\text { in days) }\end{array}$ \\
\hline $\begin{array}{c}\text { CAMPUS_TEMP } \\
(93 \%)\end{array}$ & 97.70 & 9.20 & 0.29 & 4.20 \\
\hline $\begin{array}{c}\text { OFFICE_LIGHT } \\
(97 \%)\end{array}$ & 81.45 & 9.20 & 0.35 & 11.77 \\
\hline $\begin{array}{c}\text { OFFICE TEMP } \\
(97 \%)\end{array}$ & 81.42 & 9.20 & 0.35 & 11.77 \\
\hline $\begin{array}{c}\text { CO_SENSOR } \\
(93 \%)\end{array}$ & 239.10 & 16.74 & 2.93 & 41.80 \\
\hline NOX_SENSOR & 239.10 & 16.74 & 2.93 & 41.80 \\
\hline $\begin{array}{c}\text { O3_SENSOR } \\
\underset{(92 \%)}{\text { SEN }}\end{array}$ & 239.10 & 19.13 & 2.93 & 36.57 \\
\hline $\begin{array}{c}\mathrm{NO} 2 \text { _SENSOR } \\
(92 \%)\end{array}$ & 239.10 & 19.13 & 2.93 & 36.57 \\
\hline
\end{tabular}

cycle of the ESP8266 platform (9 seconds per data sampling and sending) before compression and bringing it back to the battery capacity $(6000 \mathrm{mAh})$, the battery life for the datasets considered is between a few hours and a few days. After compression by segmentation, we change the scale for each of the considered datasets: the battery lifetimes initially in hours become in days (or weeks), and the lifetime in days become months.

\subsubsection{Sending period}

The computation of the appropriate sending period is performed accordingly to the number of user requests and thus, as long as no data is requested by the users, it is irrelevant to establish an energy intensive data connection to send the values at high frequency. Nonetheless, they can be buffered locally, e.g., in the EEPROM memory of our setup, and sent when needed. Thus, the proposed optimization does not decrease the quality of data but only the latency to obtain it. As mentioned in SEC. 4, it is the responsibility of the activity node, i.e., a node using a time-slot storage to store the number of data accesses per time slot, to store the sensor activity.

We have mocked the sending periods displayed on FIG. 17. These periods allows to feed applications that needs to monitor the temperature during daylights (time-slots 7 to 18) and more intensively around lunchtime, resulting in more requests during this period. 


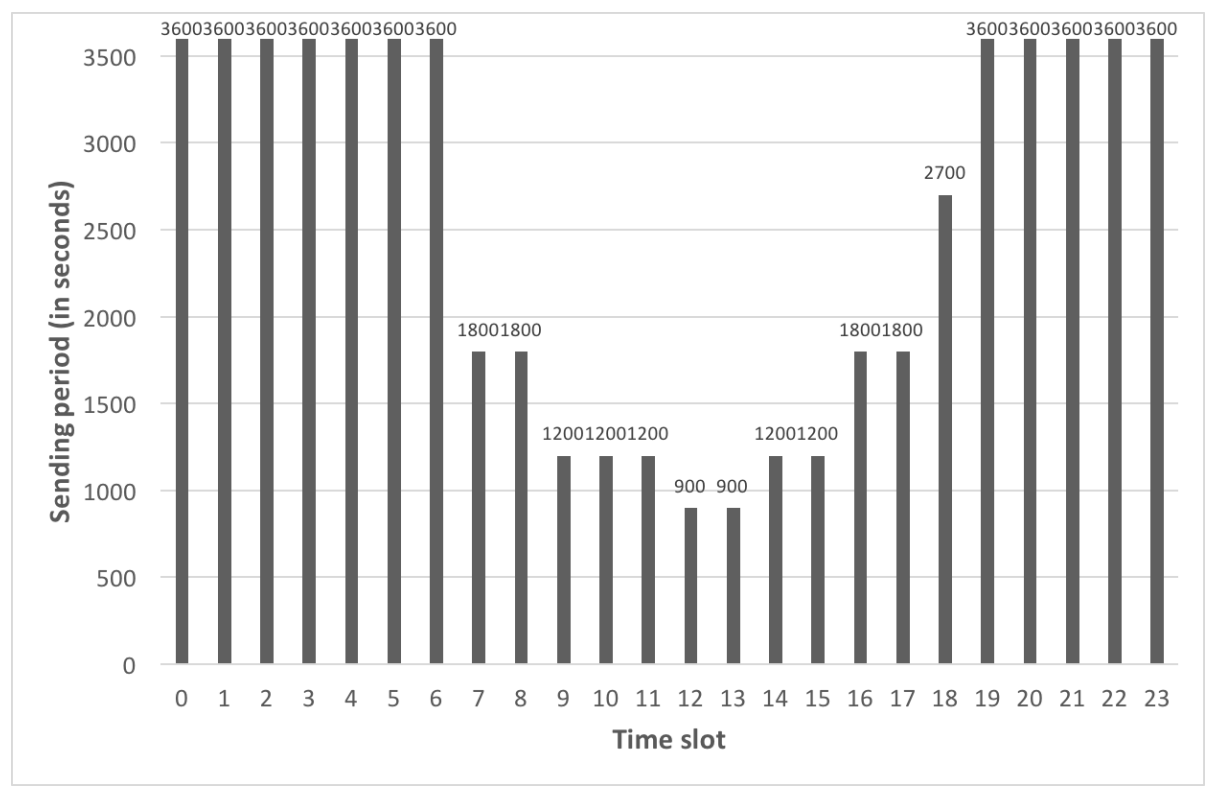

Figure 17: Computed daily sending periods (Exp.3)

For a time slot $t \in T$, the integer division of Psamp $_{t}$ with Psend $_{t}$ returns the number of data that is buffered on the platform between every sending. For example, from the periods depicted in FIG. 16 and FIG. 17, during time slot \#1, only one value will be buffered $\left(P s a m p_{1}=3600\right.$ and $P$ send $\left.d_{1}=2894\right)$ before being sent and during time slot $\# 16,39$ values will be buffered $\left(\right.$ samp $_{16}=1800$ and Psend $_{16}=46$ ) before being sent. This last value shows that 38 energy intensive network connections have been saved thanks to buffering techniques during time slot \#16.

\subsubsection{Battery discharge}

In this experiment, we have implemented the firmware described in Alg. 1 and deployed it on our prototypical platform. This platform retrieves the sampling and sending periods from the middle layer ( $c f$. FIG. 11) and follows the values depicted respectively on FIG. 16 and Fig. 17.

Using such periods, the voltage values are plotted on Fig. 18. This figure shows that, among days and unlike Exp.1 and Exp.2, the voltage value at the battery terminals remains constant. Manual measurements using a voltmeter have shown that values under $4 \mathrm{~V}$ are measurement artifacts. Comparing the battery discharge with the one observed in Exp.2 (cf. FIG. 13), we can deduce that adaptive periods allow us to extend the battery lifetime of our prototyping platform on a year scale. 


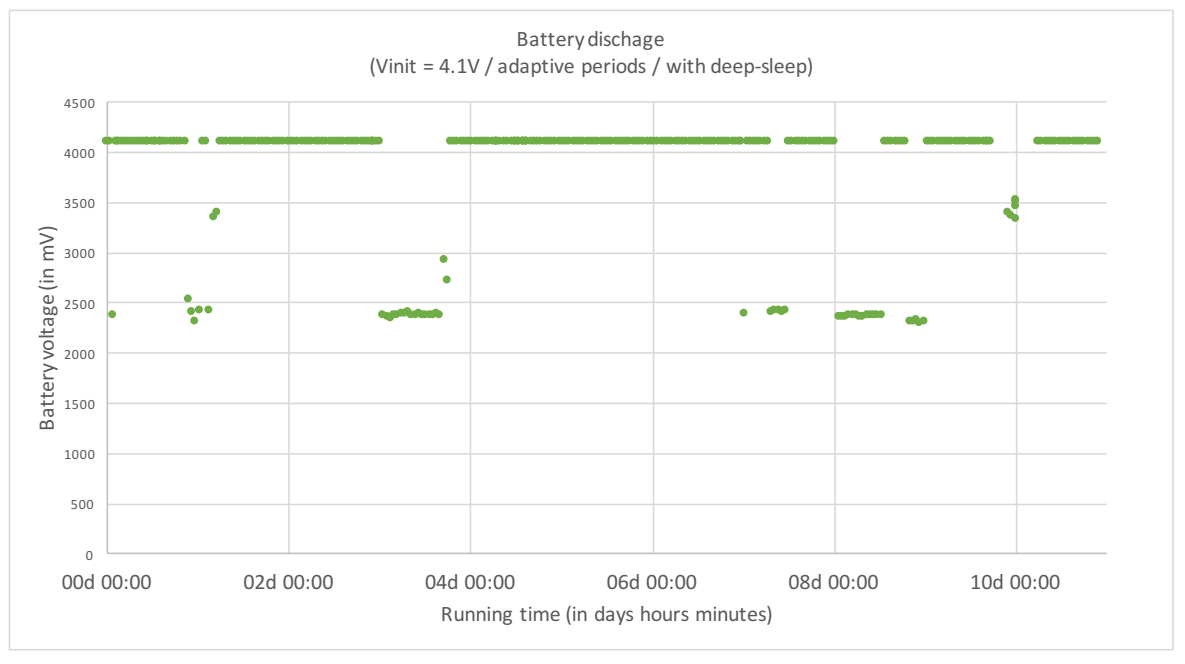

Figure 18: Battery discharge using adaptive periods (Exp.3)

\subsection{Summary}

In this section, we have conducted an experimental validation to quantify the gain in battery lifetime brought about by the use of adaptive periods to answer the first part of the research question. For each experiment, we have measured the voltage at the battery terminals and compared the obtained values with respect to methods using static periods (with or without deep-sleep). We have seen that each of these experiments allowed us to change the battery lifetime scale: a weekly scale for fixed periods without deep-sleep, a monthly scale for fixed periods with deep-sleep, and finally an yearly scale for adaptive periods with deep-sleep. Moreover, the last experiment allows us to assess the accuracy of waking-up time when the sensor platform is in deep-sleep mode. We consider the time inaccuracy to be negligible in terms of impact on the battery lifetime.

We consider these experiments to be relevant enough for measuring the gain in battery lifetime associated with the use of adaptive periods since we have compared three modes of operation: (i) fixed sending and samplig periods without deep-sleep, (ii) fixed sending and sampling periods with deep-sleep and (iii) dynamic periods with deep-sleep. These experiments can be replicated on other platforms, provided they respect the prerequisites discussed in SEC. 4.

\subsection{Threats to validity}

Regarding measurements, voltage values were acquired by measuring the voltage at the battery terminals. These measures may be imprecise and must be considered noisy if taken as a whole. However, our study aims to measure a trend (increase or decrease) in battery life, which nuances this imprecision. 
In addition, we have regularly confirmed the measured values with a digital multi-meter, confirming that the values obtained are close to reality.

Besides, some platforms integrate co-processors dedicated to specific functions (e.g., data acquisition) and designed to be energy efficient. For example, ESP platforms integrate ULP ${ }^{12}$ (Ultra Low Power) coprocessors designed to perform measurements using an ADC (Analog-Digital Converter), a temperature sensor and external sensors, while the main processor is in deep sleep mode. Our approach considers only the main processor and could be extended to benefit from these co-processors for the sampling. We expect this extension to significantly increase the battery lifetime gain obtained in our experiments. Conversely, badly designed deep-sleep modes and attached devices can overuse the battery and leak unnecessary energy limiting the gains made by activating deep-sleep mode and thus, the gains made by our proposed approach. Our contribution obviously exposes advantages and drawbacks of only interacting with devices through their deep-sleep mode interface. Still these devices are expected to well design their platform with regards to this mode.

Regarding the polynomial computation and re-training, these steps can affect system performance since it involves crossing the graph to retrieve the compressed node, read the current value and potentially update the polynomial or create a new timepoint if a significant change in data has been detected. To takle this issue, the middlelayer uses the GreyCat framework for storage and online learning. The evaluation of this framework performed in [30] showed read and write throughputs close to 100,000 nodes per second and a modify throughput close to 400,000 nodes per second. Hence, since a single physical sensor is described by three nodes (sensor node, activity node and compressed node, $c f$. SEC. 4.2), the approach can theoretically scale up to tens of thousands of sensors while having a re-training time of a few tens of millisseconds.

\section{Related Work}

In this work, we propose a cross-layer solution to reduce the energy consumption of a sensor platform. On the sensor platform layer, we rely on dynamic periods to sample and access the network. On the application layer, we rely on live machine learning techniques in order to not solicit the sensor network when values can be predicted. We have already introduced live machine learning in SEC. 2. In this section and with respect to our contributions, we compare in a first part, works addressing the dynamic periods problem before, in a second part, addressing MAC protocol optimizations and software-defined power meters. Then, we discuss machine learning techniques for forecasting data.

Dynamic periods. Dynamic periods can reduce the energy consumption by sampling or sending sensor data only when needed. The approach proposed by Kho et al. [31] provides a decentralized control of adaptive sampling. They introduce

\footnotetext{
12 http://esp-idf.readthedocs.io/en/latest/api-guides/ulp.html
} 
a metric based upon Fisher information and Gaussian process regression to define three algorithms that compute a sampling period according to the gathered information. Alippi et al. [32] propose an algorithm that dynamically estimate a sampling period according to the observed signal. With this algorithm, they can minimize the activity of both the sensor and the radio, and thus, reducing the energy consumption. A simulation of this algorithm over a snow monitoring use case shows that up to $97 \%$ of the energy can be saved. More generally, the literature provides solutions based on gathered sensor measures to compute an adaptive sampling period [33]. However, they do not focus on applications' requests. Whereas in our approach, we observe when data are requested in order to establish energy intensive network connections only when necessary.

MAC protocols. According to the IEEE 802 model, the Media Access Control layer is responsible for network access. Numerous works have been achieved to reduce the energy footprint by defining energy-efficient MAC protocols. The TMAC protocol [34] is a contention-based protocol for wireless sensor networks. It introduces an adaptive duty cycle relying on fine-grained timeouts and can save as much as $96 \%$ of the energy.

In our approach, in addition to reduce the network access with adaptive periods, we use the deep-sleep mode after every sending that shuts down the physical network interface and most of the sensor platform's functionality, saving its energy

The DMAC protocol [35] uses the tree structure describing the major traffic schema in wireless sensor networks to optimize the energy. It schedules the activity of nodes according to their depth in the tree. Therefore, all the nodes on a multi-hop path can be notified of data delivering and can send their own measurements. Various experiments on this protocol have shown an energy saving of up to 6 times greater than conventional protocols. In our approach, we define a sending period for a single period but we reusing the concepts brought by DMAC by computing inter-platform dependent sending periods is a foreseen work.

Software-defined power meter. The WattsKit approach [36] aims at using machine learning methods to monitor the energy consumption of distributed systems without the use of invasive and costly physical meters. To this end, the WattsKit approach profiles the electric consumption of some specific hardware operations such as CPU functions (e.g., TurboBoost, Hyper-threading) or device usages (e.g., Network, Disk). Based on these profiles, a model able to predict the overall hardware consumption from simple software probes is generated. During the training phase, a physical power meter is used, to obtain the real hardware power consumption and learn the expected impact of each function or device executions. Once the training phase is done, the model can be distributed over similar hardware without the need for physical meters. This model can be executed at run-time to build ultimately a software-sefined power meters that monitors the power consumption of an application (e.g., Apache ZooKeeper). 
This software-defined power meter can be integrated into a higher-level application that can make decisions based on the identified energy consumption value (e.g., pause or delay the execution if a threshold value is exceeded). If their approach allows to obtain precisely the instantaneous power of a process (in Watt), they do not target microcontroller architectures mainly used within sensor networks. In our approach, we rely on the forecasting of data to reduce the energy consumption without the use of fine-grained prediction such as WattsKit. However, as future work, it would be relevant for a sensor platform to also use such approach and adapt its behavior according to the power required by the functions used on its hosted applications.

Forecasting data. Our approach heavily relies on a prediction model to evaluate sensor value predictability. Due to our live usage of this prediction model, the ratio between its efficiency and effectiveness is key critical. For this reason we have selected polynomial functions for their excellent scalability. In [37], the authors show that polynomial functions achieve savings range from about $50 \%$ to $96 \%$, according to the acceptable error threshold. However our contribution can be extended by any other model offering similar performance tradeoffs. In particular various methods have been used for energy efficient sensor networks [38]. The Least Men Square Algorithm [39] has been proposed to predict the measured values both at the sensor platform and remote levels. A transmission is performed only if the two predicted values differ from a variable step size. Such method offers the advantage to be simple enough to be embedded on restricted hardware, however without mid-term prediction they cannot drive deep-sleep operators like our contribution. Towards such goal, time series prediction models, as performed in our approach, give goods results to reduce the energy consumption. An adaptive model selection [37] applied on different data sets shows that despite the percentage of transmitted packets varying according to the algorithm used, they remain quite close. In our approach we rely on an polynomial regression model without memory which gives the fastest results according to the tested datasets.

\section{Conclusion \& Future work}

In this paper, we have investigated whether one can significantly gain battery lifetime for a whole sensor platform using adaptive sampling and sending periods $(R Q)$.

To compute the adaptive sampling and sending periods, we have presented a self-adaptive approach using machine learning and deep-sleep to provide an optimal configuration extending the battery lifetime of a sensor platform. The generation of the optimal configuration is performed by a prediction model deployed on a middle layer located outside the sensor network. This middle layer receives sensor measures from remote sensor platforms and collect application's requests on data (as shown on FIG. 11). According to historical values and current use of the sensor network, the middle layer foresees the next data sampling and sending periods. A firmware, deployed at the sensor platform layer, 
requests the optimal configuration and buffers locally sensor data for a sending period.

Then, to measure the gain in battery lifetime, we have performed an experimental evaluation comparing the lifetime between a platform using fixed periods (with and without deep-sleep) and a platform using adaptive periods. Relying of these adaptive periods, we have successfully lowered the battery discharge. The extrapolation of the values obtained in our experiments showed us that an ESP8266 platform relying on a $6000 \mathrm{mAh}$ battery could move from a weekly scale lifetime to a yearly scale lifetime thanks to adaptive periods.

The contribution of this paper relies on a regression model in order to evaluate if sensor values can be extrapolated and to compute appropriate sampling and sending periods. The performance of this regression model directly impacts the energy reduction effect. Therefore in a future work, we plan to explore other learning methods, e.g., neural networks, and compare their efficiency towards this usage. In particular we will explore the impact of models with memory that can detect periodic patterns, such as LSTM networks [40]. In addition we also plan to work with unbounded time-slots, that describe better physical phenomena and reduce the number of computed polynomials.

Moreover, in this contribution we have optimized sensor periods independently. However, in case of sensors measuring the same phenomenon it could be highly beneficial to exploit the graph structure to generate optimal periods for a group of sensors (for instance, different sensors in a same room that provide comparable measurements). In such context, it might be interesting to synchronize the different sampling and sending values to orchestrate the measurement collection in turns, and thus not to always using the same sensors.

Finally, as many sensor platforms are nowadays available on the market with various capabilities for deep-sleep, we plan to assess our approach with different types of microcontrollers and architectures. Ultimately this would allow to classify their suitability towards such dynamic control usage.

\section{Acknowledgements}

This collaboration between Université Côte d'Azur and DataThings is supported by the Doctoral Training programme of the European Institute of Technology (EIT). The authors would also like to thanks Assaad Mowadad, Thomas Hartmann, Gregory Nain and Matthieu Jimenez for their assistance with the experimentations and for their comments that greatly improved this article.

\section{References}

[1] A. H. Ngu, M. Gutierrez, V. Metsis, S. Nepal, Q. Z. Sheng, Iot middleware: A survey on issues and enabling technologies, IEEE Internet of Things Journal 4 (1) (2017) 1-20. 
[2] S. Bandyopadhyay, M. Sengupta, S. Maiti, S. Dutta, A survey of middleware for internet of things, in: Recent Trends in Wireless and Mobile Networks, Springer, 2011, pp. 288-296.

[3] C.-F. Chiasserini, M. Garetto, Modeling the performance of wireless sensor networks, in: INFOCOM 2004. Twenty-third AnnualJoint Conference of the IEEE Computer and Communications Societies, Vol. 1, IEEE, 2004.

[4] R. Jurdak, A. G. Ruzzelli, G. M. O'Hare, Adaptive radio modes in sensor networks: How deep to sleep?, in: Sensor, Mesh and Ad Hoc Communications and Networks, 2008. SECON'08. 5th Annual IEEE Communications Society Conference on, IEEE, 2008, pp. 386-394.

[5] A. Sharma, K. Shinghal, N. Srivastava, R. Singh, Energy management for wireless sensor network nodes, International Journal of Advances in Engineering \& Technology 1 (1) (2011) 7-13.

[6] D. Lymberopoulos, A. Savvides, Xyz: a motion-enabled, power aware sensor node platform for distributed sensor network applications, in: Proceedings of the 4th international symposium on Information processing in sensor networks, IEEE Press, 2005, p. 63.

[7] E. Shih, S.-H. Cho, N. Ickes, R. Min, A. Sinha, A. Wang, A. Chandrakasan, Physical layer driven protocol and algorithm design for energy-efficient wireless sensor networks, in: Proceedings of the 7th annual international conference on Mobile computing and networking, ACM, 2001, pp. 272-287.

[8] B. Hohlt, L. Doherty, E. Brewer, Flexible power scheduling for sensor networks, in: Proceedings of the 3rd international symposium on Information processing in sensor networks, ACM, 2004, pp. 205-214.

[9] S. Bi, C. K. Ho, R. Zhang, Wireless powered communication: opportunities and challenges, IEEE Communications Magazine 53 (4) (2015) 117-125.

[10] Z. Xiao, W. Song, Q. Chen, Dynamic resource allocation using virtual machines for cloud computing environment, IEEE transactions on parallel and distributed systems 24 (6) (2013) 1107-1117.

[11] Esp8266ex datasheet.

URL https://www.espressif.com/sites/default/files/ documentation/0a-esp8266ex_datasheet_en.pdf

[12] D. J. Hand, Principles of data mining, Drug safety 30 (7) (2007) 621-622.

[13] D. A. Dickey, W. A. Fuller, Distribution of the estimators for autoregressive time series with a unit root, Journal of the American statistical association 74 (366a) (1979) 427-431.

[14] O. Anava, E. Hazan, S. Mannor, O. Shamir, Online learning for time series prediction., in: COLT, Vol. 30, 2013, pp. 172-184. 
[15] S. De Vito, E. Massera, M. Piga, L. Martinotto, G. Di Francia, On field calibration of an electronic nose for benzene estimation in an urban pollution monitoring scenario, Sensors and Actuators B: Chemical 129 (2) (2008) 750-757.

[16] C. Cecchinel, M. Jimenez, S. Mosser, M. Riveill, An Architecture to Support the Collection of Big Data in the Internet of Things, in: International Workshop on Ubiquitous Mobile Cloud (UMC'14, co-located with SERVICES'14), IEEE, Anchorage, Alaska, USA, 2014, pp. 1-8.

[17] A. Moawad, T. Hartmann, F. Fouquet, G. Nain, J. Klein, Y. Le Traon, Beyond discrete modeling: a continuous and efficient model for iot, in: Model Driven Engineering Languages and Systems (MODELS), 2015 ACM/IEEE 18th International Conference on, IEEE, 2015, pp. 90-99.

[18] M. A. M. Vieira, C. N. Coelho, D. C. da Silva, J. M. da Mata, Survey on wireless sensor network devices, in: EFTA 2003. 2003 IEEE Conference on Emerging Technologies and Factory Automation. Proceedings (Cat. No.03TH8696), Vol. 1, 2003, pp. 537-544 vol.1. doi:10.1109/ETFA.2003.1247753.

[19] G. Wenqi, W. M. HEALY, Power supply issues in battery reliant wireless sensor networks: A review, INTERNATIONAL JOURNAL OF INTELLIGENT CONTROL AND SYSTEMS 19 (1) (2014) 15-23.

[20] H. Long, Y. Liu, Y. Wang, R. P. Dick, H. Yang, Battery allocation for wireless sensor network lifetime maximization under cost constraints, in: Proceedings of the 2009 International Conference on Computer-Aided Design, ICCAD '09, ACM, New York, NY, USA, 2009, pp. 705-712. doi:10.1145/1687399.1687529.

URL http://doi.acm.org/10.1145/1687399.1687529

[21] L. Benini, G. De Micheli, Powering networks on chips: energy-efficient and reliable interconnect design for socs, in: Proceedings of the 14th international symposium on Systems synthesis, ACM, 2001, pp. 33-38.

[22] C. M. Vigorito, D. Ganesan, A. G. Barto, Adaptive control of duty cycling in energy-harvesting wireless sensor networks, in: 2007 4th Annual IEEE Communications Society Conference on Sensor, Mesh and Ad Hoc Communications and Networks, IEEE, 2007, pp. 21-30.

[23] R. R. Rout, S. K. Ghosh, Enhancement of lifetime using duty cycle and network coding in wireless sensor networks, IEEE Transactions on Wireless Communications 12 (2) (2013) 656-667.

[24] S. Hayat, N. Javaid, Z. A. Khan, A. Shareef, A. Mahmood, S. H. Bouk, Energy efficient mac protocols, in: 2012 IEEE 14th International Conference on High Performance Computing and Communication 2012 IEEE 9th International Conference on Embedded Software and Systems, 2012, pp. 1185-1192. doi:10.1109/HPCC.2012.174. 
[25] N. A. Pantazis, S. A. Nikolidakis, D. D. Vergados, Energyefficient routing protocols in wireless sensor networks: A survey, IEEE Communications Surveys Tutorials 15 (2) (2013) 551-591. doi:10.1109/SURV.2012.062612.00084.

[26] C. Wang, K. Sohraby, B. Li, M. Daneshmand, Y. Hu, A survey of transport protocols for wireless sensor networks, IEEE network 20 (3) (2006) 34-40.

[27] S. D. Muruganathan, D. C. Ma, R. I. Bhasin, A. O. Fapojuwo, A centralized energy-efficient routing protocol for wireless sensor networks, IEEE Communications Magazine 43 (3) (2005) S8-13.

[28] Z. Shelby, K. Hartke, C. Bormann, The constrained application protocol (coap), Tech. rep. (2014).

[29] O. Giustolisi, A. Doglioni, D. Savic, B. Webb, A multi-model approach to analysis of environmental phenomena, Environmental Modelling \& Software 22 (5) (2007) 674-682.

[30] T. Hartmann, F. Fouquet, M. Jimenez, R. Rouvoy, Y. Le Traon, Analyzing complex data in motion at scale with temporal graphs, in: The 29th International Conference on Software Engineering \& Knowledge Engineering (SEKE'17), KSI Research, 2017, p. 6.

[31] J. Kho, A. Rogers, N. R. Jennings, Decentralized control of adaptive sampling in wireless sensor networks, ACM Trans. Sen. Netw. 5 (3) (2009) 19:1-19:35. doi:10.1145/1525856.1525857.

URL http://doi.acm.org/10.1145/1525856.1525857

[32] C. Alippi, G. Anastasi, C. Galperti, F. Mancini, M. Roveri, Adaptive sampling for energy conservation in wireless sensor networks for snow monitoring applications, in: 2007 IEEE International Conference on Mobile Adhoc and Sensor Systems, 2007, pp. 1-6. doi:10.1109/MOBHOC.2007.4428700.

[33] S. Siddiqui, S. Ghani, Towards dynamic polling: Survey and analysis of channel polling mechanisms for wireless sensor networks, in: 2016 International Conference on Intelligent Systems Engineering (ICISE), 2016, pp. 356-363. doi:10.1109/INTELSE.2016.7475149.

[34] T. van Dam, K. Langendoen, An adaptive energy-efficient mac protocol for wireless sensor networks, in: Proceedings of the 1st International Conference on Embedded Networked Sensor Systems, SenSys '03, ACM, New York, NY, USA, 2003, pp. 171-180. doi:10.1145/958491.958512.

URL http://doi.acm.org/10.1145/958491.958512

[35] G. Lu, B. Krishnamachari, C. S. Raghavendra, An adaptive energy-efficient and low-latency mac for data gathering in wireless sensor networks, in: 18th International Parallel and Distributed Processing Symposium, 2004. Proceedings., 2004, pp. 224-. doi:10.1109/IPDPS.2004.1303264. 
[36] M. Colmant, P. Felber, R. Rouvoy, L. Seinturier, WattsKit: SoftwareDefined Power Monitoring of Distributed Systems, in: F. Capello, G. Fox, J. Garcia-Blas (Eds.), 17th IEEE/ACM International Symposium on Cluster, Cloud and Grid Computing (CCGrid), Proceedings of the 17th IEEE/ACM International Symposium on Cluster, Cloud and Grid Computing (CCGrid), IEEE, Madrid, Spain, 2017, p. 10.

URL https://hal.inria.fr/hal-01439889

[37] Y.-A. Le Borgne, S. Santini, G. Bontempi, Adaptive model selection for time series prediction in wireless sensor networks, Signal Processing 87 (12) (2007) 3010-3020.

[38] G. Anastasi, M. Conti, M. D. Francesco, A. Passarella, Energy conservation in wireless sensor networks: A survey, Ad Hoc Networks 7 (3) (2009) 537 - 568. doi:http://dx.doi.org/10.1016/j.adhoc.2008.06.003.

URL //www.sciencedirect.com/science/article/pii/ S1570870508000954

[39] P. S. Diniz, The least-mean-square (lms) algorithm, in: Adaptive Filtering, Springer, 2013, pp. 79-135.

[40] F. A. Gers, D. Eck, J. Schmidhuber, Applying lstm to time series predictable through time-window approaches, in: International Conference on Artificial Neural Networks, Springer, 2001, pp. 669-676. 